\title{
Binding of LncRNA-DACH1 to dystrophin impairs the membrane trafficking of Nav1.5 protein and increases ventricular arrhythmia susceptibility
}

Zhenwei Pan ( $\nabla$ panzw@ems.hrbmu.edu.cn )

Harbin Medical University https://orcid.org/0000-0002-1011-0954

Gen-Long Xue

Harbin Medical University

Yang Zhang

Harbin Medical University https://orcid.org/0000-0002-6680-3098

Jiming Yang

Harbin Medical University

Ying Yang

Harbin Medical University

Ruixin Zhang

Harbin Medical University

Desheng Li

Harbin Medical University

Tao Tian

Harbin Medical University

Xiaofang Zhang

Harbin Medical University https://orcid.org/0000-0001-9866-2018

Changzhu Li

Harbin Medical University

Xingda Li

Harbin Medical University

Jiqin Yang

Harbin Medical University

Kewei Shen

Harbin Medical University

Yang Guo

Harbin Medical University

Xuening Liu

Harbin Medical University

Guohui Yang 
Harbin Medical University

\section{Yanjie Lu}

Harbin Medical University

\section{Baofeng Yang}

Harbin Medical University https://orcid.org/0000-0002-0125-1608

\section{Article}

Keywords: LncDACH1, dystrophin, Nav1.5, ventricular arrhythmia

Posted Date: February 4th, 2021

DOI: https://doi.org/10.21203/rs.3.rs-156367/v1

License: (c) (i) This work is licensed under a Creative Commons Attribution 4.0 International License. Read Full License 


\section{Abstract}

Dystrophin is a critical interacting protein of Nav1.5 that determines its membrane anchoring in cardiomyocytes. The study aims to explore whether IncRNA-DACH1(IncDACH1) can regulate the distribution of Nav1.5 by binding to dystrophin and participate in ventricular arrhythmogenesis. LncDACH1 was confirmed to bind to dystrophin. Cardiomyocyte-specific transgenic overexpression of IncDACH1(IncDACH1-TG) reduced the membrane distribution of dystrophin and Nav1.5 in cardiomyocytes. The opposite data were collected from IncDACH1 cardiomyocyte conditional knockout (IncDACH1-CKO) mice. Moreover, increased ventricular arrhythmia susceptibility was observed in IncDACH1-TG mice in vivo and ex vivo. The conservative fragment of IncDACH1 inhibited membrane distribution of dystrophin and Nav1.5 and promoted the inducibility of ventricular arrhythmia. Upregulation of dystrophin in IncDACH1-TG mice rescued the impaired membrane distribution of dystrophin and Nav1.5. The human homologue of IncDACH1 inhibited the membrane distribution of Nav1.5 in human iPS-differentiated cardiomyocytes. Collectively, IncDACH1 regulates Nav1.5 membrane distribution by binding to dystrophin and participates in ventricular arrhythmogenesis.

\section{Introduction}

The voltage gated sodium channel mediates the 0 phase depolarizing inward sodium currents of cardiomyocytes ${ }^{1}$. The expression and function of sodium channel is regulated at multiple levels encompassing gene mutation, post-transcriptional modification, post-translational modification and protein trafficking etc ${ }^{1,2}$. The disruption of either process is arrhythmogenic and occasionally causes sudden death ${ }^{2,3}$.

The membrane targeting and localization of pore-forming subunit of sodium channel Nav1.5 was regulated by several interacting proteins such as ankyrin-G, MOG1, syntrophin and dystrophin etc ${ }^{2,4}$. Dystrophin is a cytoplasmic protein that is encoded by duchenne muscular dystrophy (DMD) gene ${ }^{5}$. It distributes mainly on the cellular membrane of skeletal muscle cells and cardiomyocytes, and acts as a scaffold for Nav1. $5^{6}$. In cardiomyocytes, dystrophin controls the expression and membrane anchoring of Nav1.5. Gavillet et al. showed that knockout of dystrophin in cardiomyocytes reduced peak sodium current, Nav1.5 protein expression and conduction velocity in mice ${ }^{6}$. Subsequently, they confirmed that knockout of dystrophin inhibits membrane distribution of Nav1.5 due to the disruption of dystrophinsyntrophin complex ${ }^{7}$.

Long noncoding RNAs (LncRNAs) are a new class of RNAs that are more than 200 nts long and possess little protein coding property. ${ }^{8}$ LncRNAs have been shown to regulate multiple biological processes and participate in the pathogenesis of various diseases including cardiac diseases ${ }^{9}$. LncRNAs were shown to regulate cardiac electrophysiological property by altering the function of different ion channels. For example, the increased expression of IncRNA-Kcna2as in heart failure reduced $l_{\mathrm{ks}}$ and prolonged action potential duration (APD) ${ }^{10}$. LncRNA-MALAT1 enhanced arrhythmia susceptibility by suppressing $I_{\text {to }}$ and 
prolonging APD ${ }^{11}$. In a previous study, we found that IncRNA-CCRR (cardiac conduction regulatory) interacts with connexin-43 interacting protein 85(CIP85) to promote connexin-43 membrane distribution and improve the impaired cardiac conduction of failing hearts ${ }^{12}$. However, to date, it remains unknown whether and how IncRNA regulates sodium channel.

LncDACH1 is an intronic IncRNA located on the first intron of $D A C H 1$ gene $^{13}$. We previously showed that IncDACH1 impairs cardiac function by promoting the degradation of sarco-endoplasmic reticulum ATPase 2a (SERCA2a), and exacerbates cardiac ischemia injury by inhibiting Yes-associated protein 1(YAP1) mediated proliferation of neonatal cardiomyocytes ${ }^{13,14}$. During analyzing the interacting proteins of IncDACH1 identified by mass spectrometry, we found that dystrophin is one of them, which drove us to hypothesize that IncDACH1 may be a critical regulator of sodium channel Nav1.5.

Therefore, in this study we explored whether IncDACH1 regulates Nav1.5 by interacting with dystrophin. We found that IncDACH1 inhibited the membrane trafficking of Nav1.5 by binding to dystrophin, which led to reduced sodium current and increased ventricular arrhythmia susceptibility. The study highlights a novel mechanism for the regulation of sodium channel trafficking, and reveals a potential therapeutic target for sodium channel dysfunction related cardiac arrhythmias.

\section{Results}

\section{LncDACH1 binds to dystrophin and reduces Nav1.5 membrane distribution in cardiomyocytes of IncDACH1 transgenic mice}

We firstly validated the binding between IncDACH1 and dystrophin. The RNA pulldown plus immunoblot assay confirmed that IncDACH1 can successfully pulldown dystrophin (Figure 1A). Conversely, the immunoprecipitation study showed that the antibody for dystrophin precipitated IncDACH1, while the negative control IgG did not (Figure 1B).

We next explored the influence of IncDACH1 on cellular distribution of dystrophin. The western blot data showed that the total protein of dystrophin did not change, while the membrane fraction was reduced, and the cytoplasmic fraction increased in the hearts of IncDACH1-TG mice than wild-type (WT) controls (Figure 1C). Consistently, the membrane and total protein levels of Nav1.5 were reduced, while cytoplasmic Nav1.5 increased in the hearts of IncDACH1-TG mice than WT controls (Figure 1D). The reduced membrane distribution of dystrophin and Nav1.5 in the cardiomyocytes of IncDACH1-Tg mice was further confirmed by immunofluorescent staining (Figure 1E). The mRNA levels of dystrophin and SCN5A did not change (Figure 1F). We then evaluated the functional change of sodium channel. Consistent with reduction of membrane Nav1.5, the peak $I_{\mathrm{Na}}$ was significantly decreased in the ventricular myocytes of IncDACH1-TG mice than WT controls, while the kinetics of $/_{\mathrm{Na}}$ (activation, inactivation, and recovery) did not change (Figure 1G). Meanwhile, the conduction velocity was slower in the hearts of IncDACH1-TG than WT mice (Figure 1H). 
We then applied IncDACH1 adenovirus to cultured neonatal cardiomyocytes to confirm the regulation of IncDACH1 on sodium channel in vitro. Infection of adenovirus carrying IncDACH1 significantly upregulated the level of IncDACH1 (Figure $2 \mathrm{~A}$ ) and remarkably inhibited peak $/ \mathrm{Na}$ with no change in kinetics (Figure 2B, C). The membrane distribution of dystrophin and Nav1.5 was remarkably reduced by overexpression of IncDACH1 as indicated by immunofluorescent staining (Figure 2D). The mRNA levels of dystrophin and SCN5A were not altered by IncDACH1 overexpression (Figure 2E).

\section{Inhibition of IncDACH1 in cardiomyocytes increased membrane Nav1.5 distribution}

We then employed IncDACH1 cardiomyocyte conditional knockout(IncDACH1-CKO) mice to examine the regulation of IncDACH1 on dystrophin and Nav1.5. The western blot data showed that membrane distribution of dystrophin was increased in the hearts of IncDACH1-CKO mice than WT controls, while the total dystrophin protein and dystrophin mRNA did not change (Figure 3A). Consistently, the membrane and total level of Nav1.5 was increased in the hearts of IncDACH1-CKO mice than WT controls, with no change on SCN5A mRNA (Figure 3B). The change on dystrophin and Nav1.5 membrane distribution was further validated by immunofluorescent staining (Figure 3C). Meanwhile, the peak $/_{\mathrm{Na}}$ was larger in cardiomyocytes of IncDACH1-CKO mice than WT controls, while the kinetics of $I_{\mathrm{Na}}$ (activation, inactivation, and recovery) did not change (Figure 3D). Consistent with the increase of peak $/_{\mathrm{Na}}$, the conduction velocity in the hearts of IncDACH1-CKO mice was faster than WT controls (Figure 3E).

We further confirmed effects of IncDACH1 knockdown with its siRNAs on sodium channel in cultured neonatal cardiomyocytes in vitro. Infection of adenovirus carrying siRNAs for IncDACH1 significantly reduced the level of IncDACH1 (Figure 4A). The patch-clamp recordings showed that knockdown of IncDACH1 significantly increased the current density of peak $/_{\mathrm{Na}}$ with no change on channel kinetics (Figure 4B, C). Membrane distribution of dystrophin and Nav1.5 were both increased after knockdown of IncDACH1 as indicated by immunofluorescent staining (Figure 4D). The mRNA levels of dystrophin and SCN5A were not altered by IncDACH1 knockdown (Figure 4E).

\section{Transgenic overexpression of IncDACH1 is arrhythmogenic in mice}

We next evaluated whether the inhibition of Nav1.5 by IncDACH1 is arrhythmogenic in IncDACH1-TG mice. Electrical pacing technique was employed to evaluate the arrhythmia susceptibility of intact hearts in vivo and isolated hearts ex vivo. The in vivo study showed that programmed pacing induced more ventricular arrhythmia in IncDACH1-TG mice that WT controls. Both induction rate and episodes of ventricular arrhythmia were higher in IncDACH1-TG mice (Figure 5A). In consistent, the ex vivo electrical pacing study demonstrated that ventricular arrhythmia was more frequently occurred in IncDACH1-Tg mice (Figure 5B). The optimal mapping study revealed that there are more breaking points in the perfused heart of IncDACH1-TG mice than WT controls (Figure 5C-E). Conversely, no ventricular arrhythmia was induced in the hearts of IncDACH1-CKO mice (Figure 5F). 


\section{The conservative fragment of IncDACH1 reduced peak sodium current and promoted ventricular arrhythmia}

The sequence blasting data showed that the fragment of IncDACH1 from 835 to $2085 \mathrm{nts}$ is conservative between human and mouse. We then cut IncDACH1 into different fragments (Figure 6A) to evaluate the sequence that is responsible for the binding with dystrophin. The data showed that only fragments containing the conserved sequence, fragments $a$ and $b$, can pulldown dystrophin, indicating that the conserved sequence may be the functional region of IncDACH1 (Figure 6A).

We then examined the influence of the conserved sequence from 835 to $2085 \mathrm{nts}$ (conserved fragment of IncDACH1, cF-IncDACH1) on cardiac sodium channel. The adenovirus carrying cF-IncDACH1 was constructed and administered to mice. The successful overexpression of cF-IncDACH1 was validated by qRT-PCR (Figure 6B). Administration of cF-IncDACH1 reduced the membrane distribution, and increased cytoplasmic expression of both dystrophin and Nav1.5 as indicated by western blot and immunofluorescent data (Figure 6C, D). The mRNA levels of dystrophin and Nav1.5 were not affected by cF-IncDACH1 (Supplemental Figure 1A). Overexpression of cF-IncDACH1 reduced peak $I_{\mathrm{Na}}$ currents (Figure 6E), and produced no influence on channel kinetics (Supplemental Figure 1B). The optical mapping data showed that administration of $\mathrm{CF}-\mathrm{InCDACH} 1$ reduced conduction velocity and increased break points of ventricular arrhythmias (Figure 6F, G). The induction rate and episodes of ventricular tachycardia (VT) were higher in cF-IncDACH1 group than controls (Figure $6 \mathrm{H}$ ).

In cultured neonatal cardiomyocytes, overexpression of $\mathrm{cF}-\mathrm{InCDACH} 1$ reduced peak $/ \mathrm{Na}$ with no change in kinetics, inhibited membrane distribution of dystrophin and Nav1.5, and produced no influence on the mRNA levels of dystrophin and SCN5A (Supplemental Figure 2).

\section{Activation of dystrophin transcription by dCas9-SAM system prevented the reduction of sodium current in IncDACH1 transgenic mice}

As IncDACH1 reduced Nav1.5 membrane targeting by interacting with dystrophin, we reasoned that overexpression of dystrophin may rescue the inhibition of Nav1.5 by IncDACH1. To test this notion, we constructed the AAV9 virus carrying dCas9-SAM system that can activate dystrophin transcription (AAV9Dys-Act) to perform rescuing experiments on IncDACH1-TG mice (Supplemental Figure 3). Tail vein injection of AAV9-Dys-Act virus significantly increased the mRNA level of dystrophin in the hearts of both WT and IncDACH1-TG mice (Figure 7A). The western blot data showed that overexpression of dystrophin with AAV9-Dys-Act virus increased both total and membrane protein expression of dystrophin, and rescued the reduction of dystrophin expression in InDACH1-Tg mice (Figure 7A). The mRNA level of SCN5A was not influenced by AAV9-Dys-Act virus (Figure 7B). Strikingly, AAV9-Dys-Act virus administration restored total and membrane expression of Nav1.5 in InDACH1-TG mice (Figure 7B). In addition, AAV9-Dys-Act virus injection rescued the reduction of peak $/_{\mathrm{Na}}$ current in InDACH1-TG mice (Figure $7 \mathrm{C}$ ). The kinetics of $I_{\mathrm{Na}}$ current did not change among groups (Figure $7 \mathrm{C}$ ). Activation of dystrophin transcription with AAV9-Dys-Act virus restored the conduction velocity in IncDACH1-TG mice (Figure 7D). 
Both in vivo and ex vivo data indicated that activation of dystrophin transcription reduced the susceptibility to ventricular arrhythmia of IncDACH1-TG mice (Figure 7E, F).

\section{Hadhb binds to IncDACH1 and promotes its decay}

Reduced Nav1.5 expression and reduction of peak $/_{\mathrm{Na}}$ in heart failure have been reported by a bunch of studies $^{15,16,17,18}$. We therefore evaluated the contribution of IncDACH1 on sodium channel remodeling in transaortic constriction (TAC) induced heart failure model in mice. We found that IncDACH1 was increased in failing hearts than sham controls (Figure $8 \mathrm{~A}$ ). Although IncDACH1 was upregulated in failing hearts, the mRNA of its host gene DACH1 did not change (Figure $8 \mathrm{~B}$ ). This finding excluded the transcription related mechanism on IncDACH1 upregulation during heart failure. By analyzing the RNA Pulldown plus Mass Spectrometry data, we identified three potential interacting proteins of IncDACH1 that have been shown to regulate RNA stability. They are ANP32a (acidic leucine-rich nuclear phosphoprotein 32A), elF4A1 (eukaryotic initiation factor 4A1) and hydroxyacyl-CoA dehydrogenase subunit beta (hadhb). We therefore speculated that it may be the change of RNA stability that renders to the expression change of IncDACH1. We then tested whether these proteins can affect IncDACH1 level by knocking down their expression with siRNA. The data showed that knockdown of hadhb increased the expression of IncDACH1, while knockdown of ANP32a and elF4A1 produced no influence (Figure 8C). The influence of hadhb on IncDACH1 stability was further validated by the fact that knockdown of hadhb increased the decaying half-life of IncDACH1 (Figure 8D). Furthermore, the sense sequence of IncDACH1 successfully pulled down hadhb, and the antibody of hadhb precipitated IncDACH1(Figure 8E).

Additionally, the protein level of hadhb was reduced in mouse failing hearts (Figure 8F), which is inversely correlated to the upregulation of IncDACH1. The siRNA for hadhb reduced the expression of Nav1.5 (Figure $8 \mathrm{G}$ ). These data indicated that hadhb is an upstream regulator of IncDACH1 which determines the stability of IncDACH1.

We lastly evaluated the human conserved sequence of IncDACH1(hcF-IncDACH1) on Nav1.5 distribution of human iPS induced cardiomyocytes. We found that overexpression of hcF-IncDACH1 reduced the membrane distribution of dystrophin and Nav1.5 (Figure 8H).

\section{Discussion}

In this study, we discovered that IncDACH1 is critical regulator of sodium channel in the heart. LncDACH1 binds to dystrophin and thus inhibits membrane trafficking of Nav1.5, which leads to the reduction of peak sodium current and impairment of cardiac conduction. Therefore, upregulation of IncDACH1 increased the susceptibility to ventricular arrhythmia (Fig 8I).

LncRNAs have been established to be critical regulators of various biological processes ${ }^{19}$. The action modes of IncRNAs are complex. One major mechanism for them to exert their biological function in the cytoplasm is to interact with the molecules such as proteins, miRNAs, mRNAs to alter protein translation, enzyme activity, protein degradation, etc ${ }^{20}$. For instance, IncRNA- CCRR was shown to inhibit the 
endocytic trafficking of connexin- 43 by binding to CIP85 ${ }^{12}$. LncDACH1 mainly distributes in the cytoplasm, and can bind to SERCA2a to promote its ubiquitination and degradation ${ }^{13}$. LncDACH1 can also bind to protein phosphatase 1 catalytic subunit alpha (PP1A) to inhibit its dephosphorylation activity on yes-associated protein 1 (YAP1), leading to the cytoplasmic sequestration of YAP1 ${ }^{14}$. The unraveling of the molecular mechanism of Nav1.5 expression and function is critical for the insightful understanding of sodium channel function under physiological and pathological conditions. Several interacting proteins have been demonstrated to determine membrane distribution of Nav1.5 and sodium channel function ${ }^{4}$. Dystrophin is a well-characterized Nav1.5 partner protein. It indirectly interacts with Nav1.5 via syntrophin, which binds with the C-terminus of dystrophin and with the SIV motif on the Cterminus of Nav1.56, 21. In this study, we found that IncDACH1 binds to dystrophin and leads to the impairment of Nav1.5 trafficking and reduced membrane distribution. This is so far the first evidence verifying the regulation of IncRNA on cardiac sodium channel.

Although the membrane distribution of both dystrophin and Nav1.5 was inhibited by IncDACH1, the total protein level of dystrophin was not affected, while Nav1.5 was reduced. The mechanism for the differential influence of IncDACH1 on total protein levels of dystrophin and Nav1.5 is unclear. One explanation may be that Nav1.5 is a membrane channel protein. If they failed to target on the plasma membrane, they may be regarded as unnecessary protein and undergo the process of protein degradation. The E1-E3 enzymes in the ubiquitination systems have been shown to regulate the degradation of Nav1.5, which includes E1 enzyme UBE1(Ubiquitin-activating Enzyme1), UBA6(Ubiquitinlike modifier-activating enzyme 6), E2 enzyme, UBC9 (Ubiquitin-Conjugating Enzyme 9), and E3 ligase Nedd4-2 (neuronal precursor cell expressed developmentally downregulated 4-2) $22,23,24$. LITAF (lipopolysaccharide-induced tumor necrosis factor-alpha factor), a protein encoding a regulator of endosomal trafficking, was shown to reduce surface Nav1.5 by promoting degradation of NEDD4-2 ${ }^{25}$. Therefore, cytoplasmic Nav1.5 that failed to target on plasma membrane may be quickly distinguished and then degraded by these ubiquitination enzymes.

The dysfunction of sodium channel is associated with various arrhythmias. Reduced peak $/_{\mathrm{Na}}$ due to SCN5A loss-of-function mutation can cause a series of arrhythmias such as atrial fibrillation, Brugada syndrome, long QT syndrome, sudden cardiac death, and ventricular tachycardia etc ${ }^{26,27}$. Consistently, we found that accompanied with the reduction of peak $N_{\mathrm{Na}}$, overexpression of $\mathrm{IncDACH} 1$ reduced ventricular conduction velocity and increased the susceptibility to ventricular arrhythmia in mice. The increased peak $/_{\mathrm{Na}}$ due to SCN5A gain-of-function mutation is associated arrhythmias such as atrial fibrillation, long QT syndrome; polymorphic ventricular complexes and ventricular arrhythmia ${ }^{26,27}$. In this study, although the peak $/_{\mathrm{Na}}$ increased in IncDACH1-CKO mice, the susceptibility to arrhythmia did not increase. One difference of our data with SCN5A gain-of-function mutation is that the kinetics of peak $I_{\mathrm{Na}}$ often change during mutation, while it is not the case of IncDACH1 knockout. One possible explanation may be that the increase of peak $I_{\mathrm{Na}}$ has not reach the limit to break the electrophysiological balance of the heart. 
Sodium channel remodeling is commonly occurred in cardiac diseases especially heart failure. Despite of some discrepancy, the main observations are that the peak $/_{\mathrm{Na}}, \mathrm{SCN} 5 \mathrm{~A}$ mRNA and Nav1.5 protein are reduced during heart failure of both human patients and animal models $15,16,17,18$. In this study, we found that IncDACH1 was increased during heart failure, indicating that it may contribute to sodium channel remodeling and arrhythmogenesis during heart failure by interfering the action of dystrophin. Interestingly, we found that activation of dystrophin with dCas9-SAM system restored the membrane distribution of Nav1.5 in cardiomyocytes of IncDACH1-TG mice, which implies its potential in counteracting sodium channel remodeling of patients with heart failure.

The low sequence conservation of IncRNAs among species is a critical issue that limits the extrapolation of data from animal studies to human beings ${ }^{28,29}$. In this study, we found that the conserved fragment of IncDACH1 exhibits the same effect as IncDACH1 on Nav1.5 trafficking and arrhythmogenesis. Moreover, the human conservative homologous fragment of IncDACH1 can inhibit the membrane distribution of Nav1.5 in cardiomyocytes derived from induced pluripotent stem cells (iPS-CMs). These findings hint the clinical relevance of IncDACH1, which holds the potential to become a therapeutic target for treating sodium channel remodeling in clinic.

In conclusion, IncDACH1 is a novel regulator of sodium channel, which suppresses the membrane trafficking of Nav1.5 by disturbing the function of dystrophin. The current work enriched our understanding of the biology of sodium channel trafficking and function, and indicated that IncRNAs possess the potential to become therapeutic targets for ventricular arrhythmias.

\section{Methods}

\section{Animals}

Neonatal (within 3-day post born) and adult C57BL/6 mice (8 to 10 weeks old) were provided by the animal center at the Second Affiliated Hospital of Harbin Medical University. Use of animals was approved by the Ethic Committees of Harbin Medical University and conformed to the Guide for the Care and Use of Laboratory Animals published by the US National Institutes of Health (NIH Publication No. 8523, revised 1996).

\section{Neonatal cardiomyocytes preparation}

Neonatal cardiomyocytes were isolated from 3-day-old mice in accordance with the following procedures. Briefly, after dissection, hearts were washed and minced in $0.25 \%$ trypsin. Pooled cell suspensions were centrifuged and resuspended in Dulbecco's modified Eagle's medium (DMEM Hyclone, USA) supplemented with $10 \%$ fetal bovine serum, $100 \mathrm{U} / \mathrm{ml}$ penicillin and $100 \mu \mathrm{g} / \mathrm{ml}$ streptomycin. The suspension was incubated in culture flasks for $90 \mathrm{~min}$, which makes fibroblasts preferentially adhere to the bottom of the culture flasks. Neonatal cardiomyocytes were removed from the culture flasks and the medium was changed. Cell cultures were incubated for $48 \mathrm{~h}$ at $37^{\circ} \mathrm{C}$ in a humidified atmosphere of $95 \%$ oxygen and $5 \%$ carbon dioxide before any experimentation. 
Generation of cardiac myocyte-specific IncDACH1 overexpressing mice

Cardiomyocyte-specific IncDACH1 overexpressing mice driven by murine aMHC promoter on a C57BL/ 6 background was generated by Biocytogen Co., Ltd (Beijing, China) as demonstrated in previous study ${ }^{13}$.

\section{Generation of cardiomyocyte-specific IncDACH1 knockout mice}

LncDACH1 conditional KO mice (IncDACH1 Flox/Flox) was generated by using CRISPR/Cas9 technique on C57BL/ 6 background mice by Biocytogen Co., Ltd (Beijing, China) as demonstrated in previous study ${ }^{13}$.

Construction of adeno-associated virus 9 (AAV9) carrying deactivated clustered regularly interspaced short palindromic repeats associated protein 9 nuclease- synergistic activation mediator(dCas9-SAM) system to activate the transcription of dystrophin

Adeno-associated virus 9(AAV9) carrying dCas9-SAM system to activate the transcription of dystrophin was constructed as reported previously with brief modifications ${ }^{30}$. The sgRNA targeting on the promoter region of dystrophin was designed and cloned into the multiple cloning site of plasmid GV639 (EFS-NLSdSaCas9-NLS-VP64-bGHpA-U6). The constructed plasmid was packaged into AAV9 virus. The sequence of sgRNA is: 5'- CGCTTCCGCGGCCCGTTCAA -3'; The mock-sgRNA target sequence (5'-

CGCTTCCGCGGCCCGTTCAA - $3^{\prime}$ ) was used as negative control. The obtained AAV9 virus volume was administered into C57BL/ 6 mice via tail vein injection at $1 \times 10^{11}$ genome containing particles

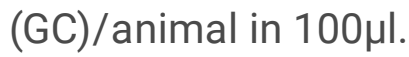

\section{Construction of adenovirus carrying cF-IncDACH1 and in vivo gene delivery}

Adenovirus vectors carrying cF-IncDACH1 (OE- cF-IncDACH1) and a negative control (NC) and a CAG promoter conjugated with green fluorescent protein (GFP) were constructed by Genechem Co., Ltd. (Shanghai, China). OE- CF-IncDACH1, control constructs at $1 \times 10^{9}$ genome containing particles (GC)/animal in $100 \mu$ l volume was administered into C57BL/ 6 mice with body weights ranging from $18 \sim 22 \mathrm{~g}$ via tail vein injection. Seventy-two hours after injection, the mice were subjected to further study.

\section{Construction of adenovirus carrying IncDACH1, IncDACH1 siRNA, conserved fragment of human} IncDACH1 and infection

Adenovirus vectors carrying IncDACH1 (OE-IncDACH1), a short RNA fragment for silencing IncDACH1 (SiIncDACH1) or conserved fragment of human IncDACH1 (hcF-IncDACH1) and a CAG promoter conjugated with green fluorescent protein (GFP) were constructed by Genechem Co., Ltd. (Shanghai, China). Neonatal cardiomyocytes were infected with adenovirus for 48 hours, and then subjected to subsequent study.

Transfection of Hadhb, Eif4a1, and Anp32 siRNA. 
siRNA for Hadhb (siHadhb), Eif4a1(siEif4a1), Anp32(siAnp32) and a scrambled negative control RNA (siNC) were synthesized by Generalbiol (Chuzhou, Anhui, China). These siRNAs were transfected at a final concentration of $100 \mathrm{nM}$ into NMVMs using the X-treme GENE Transfection Reagent (Roche, Indianapolis, USA) according to the manufacturer's protocols. The cardiomyocytes were collected for total RNA isolation or protein purification. The sequences are: siHadhb, sense 5'- GCUCUCAGAUCUUCUAUAATT-3' and antisense 5'- UUAUAGAAGAUCUGAGAGCTT-3'; siEif4a1, sense 5'- GCUCACCGAGAAGAUGCAUTT-3' and antisense 5'- UUAUAGAAGAUCUGAGAGCTT-3'; siAnp32, sense 5'- GCCUCACCUCCAUUUCCAATT-3' and antisense 5'- UUGGAAAUGGAGGUGAGGCTT-3'.

\section{Induction of ventricular arrhythmia}

C57BL/6 mice were anesthetized with 2,2,2-tribromoethanol (200 mg/kg, i.p.). An octapolar electrophysiological catheter (1.1F, SciSense Inc., Canada) was inserted into the right ventricle via the jugular vein. Intracardiac pacing was performed using an automated stimulator interfaced with the data acquisition system (GY6000; HeNan HuaNan Medical Science \& Technology Ltd., Zhengzhou, China). The surface recording electrode was fixed on LV epicardium to record pseudo-ECG. Inducibility of ventricular tachycardia (VT) was determined by applying a train of ten consecutive electrical pulses with a coupling interval of $80 \mathrm{~ms}$ (S1), followed by two extra stimuli (S2 and S3) at coupling intervals of $2 \mathrm{ms,}$ respectively. Successful induction of VT was defined as the appearance of rapid nonsinus rhythm ventricular activations lasting for three beats or more.

\section{Optical mapping recording}

Mice were heparinized and euthanized by 2,2,2-tribromoethanol $(200 \mathrm{mg} / \mathrm{kg}$, intraperitoneal injection; Sigma, St Louis, MO, USA). The heart was isolated and langendorff perfused with Tyrode's solution $(\mathrm{NaCl}$ $128.2 \mathrm{mM}, \mathrm{CaCl}_{2} \cdot 2 \mathrm{H}_{2} \mathrm{O} 1.3 \mathrm{mM}, \mathrm{KCl} 4.7 \mathrm{mM}, \mathrm{MgCl}_{2} \cdot 6 \mathrm{H}_{2} \mathrm{O} 1.85 \mathrm{mM}, \mathrm{NaH}_{2} \mathrm{PO}_{4} \cdot 2 \mathrm{H}_{2} \mathrm{O} 1.19 \mathrm{mM}, \mathrm{Na}_{2} \mathrm{CO} 320$ $\mathrm{mM}$, and glucose $11.1 \mathrm{mM}$; pH 7.35) at $37^{\circ} \mathrm{C}$. After 10 min of stabilization, the hearts were stained with $\mathrm{RH} 237(10 \mu \mathrm{M})$ for membrane voltage $(\mathrm{Vm})$ mapping. The dye was excited at $710 \mathrm{~nm}$ using monochromatic light-emitting device. The fluorescence was filtered and recorded simultaneously with a MiCAM05 CMOS camera (SciMedia, USA) at 1ms/frame and 100x100 pixels with a spatial resolution of $0.35 \times 0.35 \mathrm{~mm}^{2}$ per pixel. Blebbistatin $(10 \mu \mathrm{M}$, Selleckchem, Houston, TX, USA) was used to inhibit motion artifact during optical mapping.

\section{Experimental protocol of optical mapping}

A pair of hook bipolar electrodes was inserted into apex of heart for pacing. A pseudo-ECG was obtained with widely spaced bipolar electrodes to determine ventricular rhythm. The ventricles were initially paced at a constant pacing cycle length (PCL) of $200 \mathrm{~ms}$. The PCLs were progressively shortened $(200,100,60$, $40,30,20 \mathrm{~ms}$ ) with a duration of 1-2 s until ventricular tachycardia (VT) was induced or the loss of 1:1 capture of the ventricles. Optical recording was performed after 20 beats of stable pacing at each PCL. Optical recordings were then performed during VT. 


\section{Patch-clamp recording}

Whole-cell configuration of the patch-clamp technique was used to record peak $/_{\mathrm{Na}}$. Experiments were performed at room temperature $\left(22 \sim 23^{\circ} \mathrm{C}\right)$ by using a MultiClamp $700 \mathrm{~B}$ (Alembic Instruments) amplifier. Pipettes (tip resistance 1 to $2 \mathrm{M} \Omega$ ) were filled with a solution containing (in $\mathrm{mM}$ ): $\mathrm{NaCl} 5, \mathrm{CaCl}_{2}$ 2, $\mathrm{MgCl}_{2} 2$, CsCl 130, HEPES 10, EGTA 15 and MgATP 4 (pH 7.2 with CsOH). Myocytes were bathed with a solution containing (in mM): $\mathrm{NaCl} 25, \mathrm{CaCl}_{2} 2, \mathrm{MgCl}_{2} 2.5, \mathrm{CsCl} 108.5, \mathrm{HEPES} 10, \mathrm{CoCl}_{2} 2.5$ and glucose 10 (pH 7.4 with $\mathrm{CsOH})$.

\section{Differentiation of human induced pluripotent stem cells(hiPSCs) to cardiomyocytes}

Undifferentiated hiPS cells (AC-iPSC) were cultured on Matrigel-coated plates in an E8 medium (CA1001500, CELLAPY). Differentiation basal medium composed of RPMI1640 medium (C11875500BT, Thermo Fisher Scientific) and B27 minus insulin (A1895601, Thermo Fisher Scientific) was used to induce cardiomyocyte differentiation. Specifically, the 70 80\% confluent hiPSCs were incubated in differentiation basal medium added with CHIR-99021 (HY-10182, MCE) for 1 day and Wnt-C59 (S7037, Selleck Chemicals) for 2 days. Then, the cells were cultured in RPMI1640 basal medium containing B27 (17504044, Thermo Fisher Scientific), which was replaced with fresh medium every $1 \sim 2$ days. Beating cells were observed after 8 days of differentiation induction and used for further study.

\section{Construction of truncated LncDACH1 fragment plasmids}

The sequence of IncDACH1 was divided into five fragments. The cDNA of each fragment was inserted into the pCDNA3.1, respectively. The first $417 \mathrm{nts}$ of the entire sequences was cut off and constructed as fragment-a (418-2085 nts). Another $417 \mathrm{nts}$ was cut off to generate fragment-b (835-2085 nts). The third $417 \mathrm{nts}$ was cut off to generate fragment-c (1251-2085 nts). Fragment-d is from 835-1668 nts, and fragment-e is from 835-1251 nts.

\section{Isolation of cardiac myocytes}

Hearts were rapidly excised, cannulated, and perfused with $\mathrm{Ca}^{2+}$-free Tyrode solution (in $\mathrm{mM}$ ): $\mathrm{NaCl} 137$, $\mathrm{KCl} 5.4, \mathrm{NaH}_{2} \mathrm{PO}_{4}$ 0.16, glucose 10, $\mathrm{CaCl}_{2} 1.8, \mathrm{MgCl}_{2} 0.5$, HEPES 5.0, and $\mathrm{NaHCO}_{3} 3.0$ (pH 7.4 adjusted with $\mathrm{NaOH}$ ) for 5 min. The heart was then perfused with a solution containing collagenases $B$ and $D$ (Roche) and protease XIV (Sigma) until digestion was complete. Tissue was dissociated using forceps, and dissociated left ventricular cardiomyocytes were gradually exposed to $\mathrm{Ca}^{2+}$ (from 50 to $500 \mu \mathrm{M}$ over $40 \mathrm{~min}$ ) and plated in culture chambers for further studies.

\section{Immunocytochemistry of isolated mouse ventricular myocytes.}

Cardiomyocytes were fixed for 10 min with 4\% paraformaldehyde in PBS, and then washed in PBS for 10 min (2 times). The cells were permeabilized with $0.5 \%$ Tween 20 for $30 \mathrm{~min}$. After washed out with PBS for 10 min (3 times), cardiomyocytes were incubated with primary antibodies against Nav1.5 (ASC005, 
Alomone, $1 ; 200$ ) and dystrophin (MANDYS8, SIGMA, 1;300) overnight at $4^{\circ} \mathrm{C}$. Following washout with PBS (10 min, 3 times), cells were incubated with secondary antibodies for $1 \mathrm{~h}$ and washed with PBS (10 min, 3 times). The cover slips were mounted onto frosted slides in a solution composed of $90 \%$ FluorSave Reagent (Calbiochem, La Jolla, CA, USA) and 10\% 10X PBS.

\section{Fluorescent in situ hybridization (FISH)}

In situ hybridization was performed with a Fluorescent in Situ Hybridization (FISH) Kit (RiboBio, Guangzhou, China). Briefly, isolated cardiomyocytes were fixed in $4 \%$ formaldehyde at $4^{\circ} \mathrm{C}$ for $10 \mathrm{~min}$ and dried out on the slides at room temperature (RT). The slides were rinsed and permeabilized with $0.5 \%$ Triton-100 in PBS at RT for 30 min, washed with PBS solution, and prehybridized at $37^{\circ} \mathrm{C}$ for 30 min before hybridization. The prehybridized slides were then incubated with IncRNA-probe in hybridization solution at $37^{\circ} \mathrm{C}$ for $16 \mathrm{~h}$. After hybridization, the slides were washed six times with prewarmed wash buffer and PBS solution. Finally, the slides were counterstained with DAPI and visualized using a confocal laser-scanning microscope (Zeiss 800 , Germany).

\section{Quantitative Real-time RT-PCR}

Total RNA was extracted by using Trizol reagent (Invitrogen, USA) according to manufacturer's protocol. Total RNA $(0.5 \mu \mathrm{g})$ was reverse transcribed by using the TransScript reverse transcriptase (GMO technology, Beijing) to obtain cDNA. The RNA levels were determined using SYBR Green I incorporation method on ABI 7500 fast Real Time PCR system (Applied Biosystems, USA), the expression levels of mRNA were calculated by relative quantitative $2^{-\triangle \triangle C T}$ method. The primers are: Mus IncDACH1 Forward: 5'-AAGATAGGATGTTGGGGCAG-3' Mus IncDACH1 Reverse: 5'-ACCATAGCACAAACACTTCC-3' Mus Dystrophin Forward: 5'- CGGGTTGGCTTTGAATGCTC -3' Mus Dystrophin Reverse: 5'AGTCTTTGGGTGGCTGAGTG -3' Mus DACH1 Forward:5'-AACCGCAAGAGACAGCATCG-3' Mus DACH1 Reverse: 5'-GGACAGGCCATCAGGAAACAG-3' Mus SCN5a Forward: 5'- GAAGGAACGCAGCACAGACAG-3' Mus SCN5a Reverse: 5'- CATCGCCCTTGACCCATACTA -3' Mus Hadhb Forward: 5'CCAAGAAGGCACAGGATGAAG -3' Mus Hadhb Reverse: 5'- CCAGTGAGGAAGGACGGATG -3

\section{Western blot}

The total, membrane and cytoplasmic protein samples were extracted from cardiac tissues of C57BL/6 mice for immunoblotting analysis. Total protein was collected with the treatment of RIPA lysis buffer (Beyotime, Beijing, China) and a protease inhibitor cocktail (Roche, Basel, Switzerland) at $4^{\circ} \mathrm{C}$ followed by centrifugation. Extraction of surface and cytoplasmic proteins was conducted using the Surface and Cytoplasmic Protein Reagent Kit (Cat\#P0033; Beyotime, Shanghai, China) according to the manufacturer's instructions. Protein samples were fractionated by SDS-PAGE and then transferred to PVDF membrane. The membranes were blocked in Tris-buffered saline containing $5 \%$ milk and then incubated with primary antibodies at $4^{\circ} \mathrm{C}$ overnight. The primary antibodies include anti-Nav1.5 (ASC005, Alomone, 1:200), anti-dystrophin (MANDYS8, SIGMA, 1:500). The anti- $\beta$-actin (1:20000 dilution, 66009-1Ig, Proteintech) and anti-N-cadherin antibody (Cat\#ab76011, 1:5000; Abcam, Cambridge, UK) were used 
as internal controls. Western blot bands were captured on the Odyssey Infrared Imaging System (LI-COR Biosciences, USA) and quantified with Odyssey v1.2 software by measuring the band intensity (area $x$ OD) in each group. The band intensity was normalized to the internal control. All antibodies were diluted in PBS buffer.

\section{RNA pulldown and immunoblotting}

The RNA pull-down was performed as described in the previous study ${ }^{13}$. Briefly, Biotin-labeled, full length IncDACH1 RNA and antisense RNA were prepared with the Biotin RNA Labeling Mix (Roche) and T7 RNA polymerase (Roche). Biotinylated RNAs were treated with RNase-free DNase I (Invitrogen) and purified on G-50 Sephadex Quick Spin columns (Roche). Biotinylated RNA $(17 \mu \mathrm{g})$ was heated to $65^{\circ} \mathrm{C}$ for $10 \mathrm{~min}$ and slowly cooled to $4^{\circ} \mathrm{C}$. Then the RNA was mixed with tissue extracts in pulldown buffer supplemented with tRNA $(0.1 \mu \mathrm{g} / \mu \mathrm{l})$ and incubated at $4^{\circ} \mathrm{C}$ for $2 \mathrm{~h}$. Washed Streptavidin agarose beads ( $60 \mu \mathrm{l}$, Invitrogen) were added to each binding reaction and further incubated at $4^{\circ} \mathrm{C}$ for $1 \mathrm{~h}$. Beads were washed briefly five times in pulldown buffer and boiled in SDS buffer, and the retrieved protein was visualized by immunoblotting.

\section{RNA immunoprecipitation (RIP)}

RNA immunoprecipitation (RIP) experiments were performed by using a Magna RIPTM RNA-Binding Protein Immunoprecipitation Kit (Millipore, USA) as previously reported ${ }^{13}$. Briefly, heart tissue was pieced and lysed in $220 \mu \mathrm{l}$ of lysis buffer containing protease inhibitors and RNase Inhibitor and centrifuged at $14,000 \times \mathrm{g}$ for $10 \mathrm{~min}$. The supernatants were incubated with anti-dystrophin, anti-hadhb and anti-rabbit IgG antibody for overnight at $4^{\circ} \mathrm{C}$ with gentle rotation. Protein $\mathrm{G}$ magnetic beads ( $\left.50 \mu \mathrm{l}\right)$ were added and incubated at RT with gentle rotation for $3 \mathrm{~h}$. RNA was extracted with $400 \mu$ l phenol:chloroform:isoamyl alcohol $(125: 24: 1, \mathrm{pH}=4.3)$ according to the manufacturer's instructions before quantitation by RT-qPCR.

\section{Mouse models of heart failure (HF) by transaortic constriction (TAC) and by coronary artery ligation}

Mice were randomly divided into sham and TAC groups. In each group, mice were anesthetized with 2,2,2tribromoethanol ( $200 \mathrm{mg} / \mathrm{kg}$, i.p.) for TAC model. The animal was orally intubated with 20 -gauge tube, and ventilated (mouse ventilator, UGO BASILE, Biological Research Apparatus, Italy) at the respiratory rate of 100 breaths/min with a tidal volume of $0.3 \mathrm{ml}$. The transverse aorta was constricted by a 6-0 silk suture ligature tied firmly against a 27-gauge needle between the carotid arteries. Then, the needle was promptly removed to yield a constriction of $0.4 \mathrm{~mm}$ in diameter. For sham group mice, the animals received the same procedures without aorta constriction.

\section{Statistics}

Data are expressed as mean \pm SEM. Statistical analysis was performed using unpaired Student's $t$ test or One-Way Analysis of Variance (ANOVA) followed by Tukey's post-hoc analysis. A $P<0.05$ was considered statistically different.v 


\section{Declarations}

\section{Author contributions}

Genlong Xue, YZ, JY, performed experiments, analyzed data, and prepared the manuscript. Ying Yang, RZ, $\mathrm{DL}, \mathrm{TT}, \mathrm{XZ}, \mathrm{CL}, \mathrm{XL}, \mathrm{JY}, \mathrm{KS}, \mathrm{YG}, \mathrm{XL}$ and $\mathrm{GY}$ helped perform experiments and collect data. BY and YJL oversaw the project and proofread the manuscript. ZP designed the project, oversaw the experiments and prepared the manuscript.

\section{Acknowledgements}

This work was supported by National Key R\&D Program of China (2017YFC1307404 to Z. P.), National Natural Science Foundation of China (82070344, 81870295 to Z. P. 81730012,81861128022 to B. Y.), Heilongjiang Touyan Innovation Team Program and CAMS Innovation Fund for Medical Sciences (CIFMS, 2019-12M-5-078 to B. Y.).

\section{Competing interests}

The authors declare no competing interests.

\section{Data availability}

The data that support the findings of this study and unique materials are available from the corresponding authors upon reasonable request. Source data are provided with this paper. Additional data related to this paper may be requested from the authors.

\section{References}

1. Rook MB, Evers MM, Vos MA, Bierhuizen MF. Biology of cardiac sodium channel Nav1.5 expression. Cardiovasc Res 93, 12-23 (2012).

2. Marionneau $\mathrm{C}$, Abriel $\mathrm{H}$. Regulation of the cardiac $\mathrm{Na}+$ channel $\mathrm{NaV} 1.5$ by post-translational modifications. J Mol Cell Cardio/ 82, 36-47 (2015).

3. Ruan Y, Liu N, Priori SG. Sodium channel mutations and arrhythmias. Nat Rev Cardio/ 6, 337-348 (2009).

4. Abriel H, Rougier JS, Jalife J. Ion channel macromolecular complexes in cardiomyocytes: roles in sudden cardiac death. Circ Res 116, 1971-1988 (2015).

5. Lapidos KA, Kakkar R, McNally EM. The dystrophin glycoprotein complex: signaling strength and integrity for the sarcolemma. Circ Res 94, 1023-1031 (2004).

6. Gavillet B, et al. Cardiac sodium channel Nav1.5 is regulated by a multiprotein complex composed of syntrophins and dystrophin. Circ Res 99, 407-414 (2006).

7. Petitprez S, et al. SAP97 and dystrophin macromolecular complexes determine two pools of cardiac sodium channels Nav1.5 in cardiomyocytes. Circ Res 108, 294-304 (2011). 
8. Quinn JJ, Chang HY. Unique features of long non-coding RNA biogenesis and function. Nat Rev Genet 17, 47-62 (2016).

9. Bar C, Chatterjee S, Thum T. Long Noncoding RNAs in Cardiovascular Pathology, Diagnosis, and Therapy. Circulation 134, 1484-1499 (2016).

10. Long QQ, et al. Long Noncoding RNA Kcna2 Antisense RNA Contributes to Ventricular Arrhythmias via Silencing Kcna2 in Rats With Congestive Heart Failure. J Am Heart Assoc 6, (2017).

11. Zhu P, et al. Long noncoding RNA MALAT1 downregulates cardiac transient outward potassium current by regulating miR-200c/HMGB1 pathway. J Cell Biochem 119, 10239-10249 (2018).

12. Zhang $Y$, et al. Long non-coding RNA CCRR controls cardiac conduction via regulating intercellular coupling. Nat Commun 9, 4176 (2018).

13. Cai B, et al. Long Noncoding RNA-DACH1 (Dachshund Homolog 1) Regulates Cardiac Function by Inhibiting SERCA2a (Sarcoplasmic Reticulum Calcium ATPase 2a). Hypertension 74, 833-842 (2019).

14. Cai B, et al. Targeting LncDACH1 promotes cardiac repair and regeneration after myocardium infarction. Cell death and differentiation, (2020).

15. Baba S, Dun W, Cabo C, Boyden PA. Remodeling in cells from different regions of the reentrant circuit during ventricular tachycardia. Circulation 112, 2386-2396 (2005).

16. Valdivia CR, et al. Increased late sodium current in myocytes from a canine heart failure model and from failing human heart. J Mol Cell Cardio/ 38, 475-483 (2005).

17. $\mathrm{Xi} Y$, et al. Increased late sodium currents are related to transcription of neuronal isoforms in a pressure-overload model. Eur J Heart Fail 11, 749-757 (2009).

18. Dybkova $\mathrm{N}$, et al. Differential regulation of sodium channels as a novel proarrhythmic mechanism in the human failing heart. Cardiovasc Res 114, 1728-1737 (2018).

19. Yao RW, Wang Y, Chen LL. Cellular functions of long noncoding RNAs. Nat Cell Bio/ 21, 542-551 (2019).

20. Ulitsky I, Bartel DP. lincRNAs: genomics, evolution, and mechanisms. Cell 154, $26-46$ (2013).

21. Shy $D$, et al. PDZ domain-binding motif regulates cardiomyocyte compartment-specific NaV1.5 channel expression and function. Circulation 130, 147-160 (2014).

22. Tang B, et al. UBC9 regulates cardiac sodium channel Nav1.5 ubiquitination, degradation and sodium current density. J Mol Cell Cardiol 129, 79-91 (2019).

23. van Bemmelen MX, et al. Cardiac voltage-gated sodium channel Nav1.5 is regulated by Nedd4-2 mediated ubiquitination. Circ Res 95, 284-291 (2004).

24. Hu Y, et al. Ubiquitination-activating enzymes UBE1 and UBA6 regulate ubiquitination and expression of cardiac sodium channel Nav1.5. Biochem J 477, 1683-1700 (2020).

25. Turan NN, et al. The endosomal trafficking regulator LITAF controls the cardiac Nav1.5 channel via the ubiquitin ligase NEDD4-2. J Biol Chem, (2020).

26. Han D, Tan H, Sun C, Li G. Dysfunctional Nav1.5 channels due to SCN5A mutations. Exp Biol Med (Maywood) 243, 852-863 (2018). 
27. Savio-Galimberti E, Argenziano M, Antzelevitch C. Cardiac Arrhythmias Related to Sodium Channel Dysfunction. Handb Exp Pharmacol 246, 331-354 (2018).

28. Johnsson P, Lipovich L, Grander D, Morris KV. Evolutionary conservation of long non-coding RNAs; sequence, structure, function. Biochim Biophys Acta 1840, 1063-1071 (2014).

29. Tsagakis I, Douka K, Birds I, Aspden JL. Long non-coding RNAs in development and disease: conservation to mechanisms. J Pathol 250, 480-495 (2020).

30. Maeder ML, Linder SJ, Cascio VM, Fu Y, Ho QH, Joung JK. CRISPR RNA-guided activation of endogenous human genes. Nat Methods 10, 977-979 (2013).

\section{Figures}


A

C

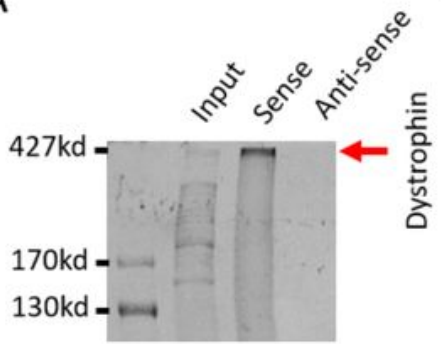

Dystrophin $\frac{\text { WT }}{\beta \text {-actin }}$

Dystrophin $\frac{W T}{-T G}$
N-cadherin

Dystrophin $\underline{\text { WT }}$
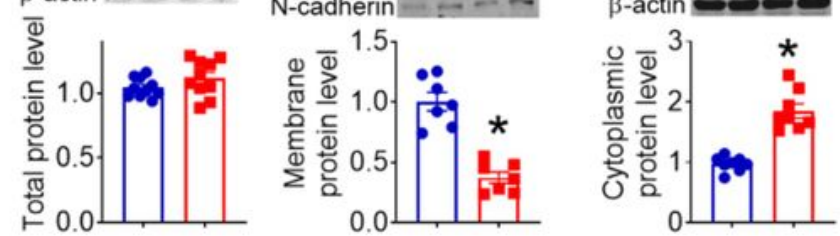

B
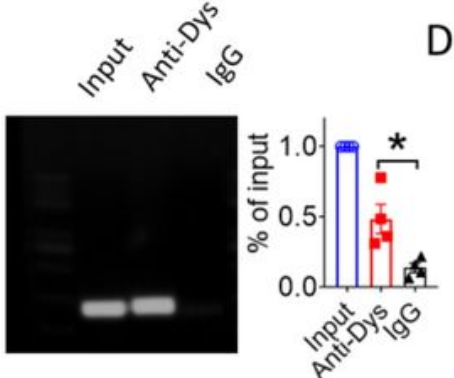

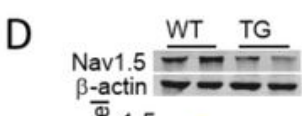
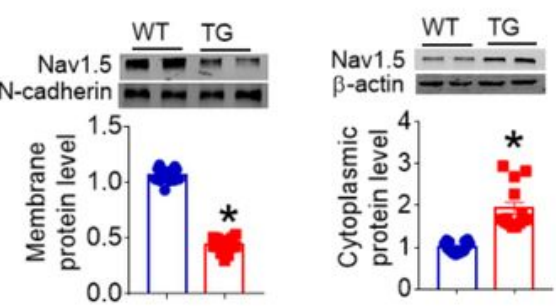

든

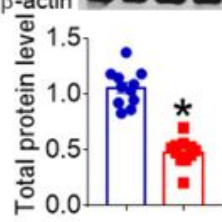

$\mathrm{N}$-cadherin $-\infty \mathrm{m}=$

$\beta$-actin $=-\div-$

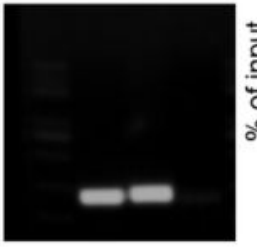

مै:

$\mathrm{E}$

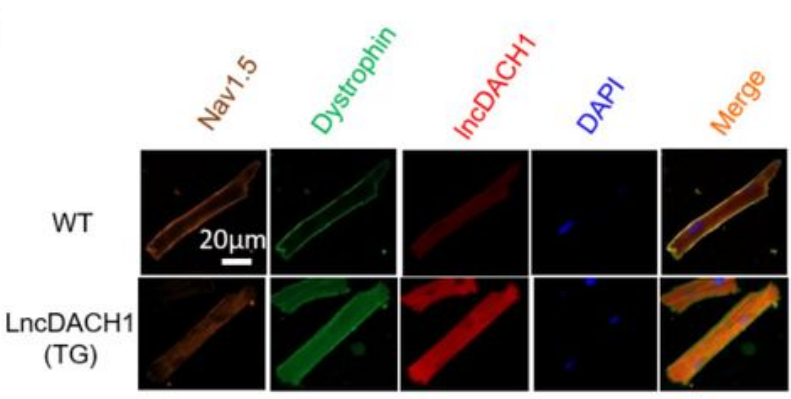

F

Dystrophin mRNA SCN5A mRNA
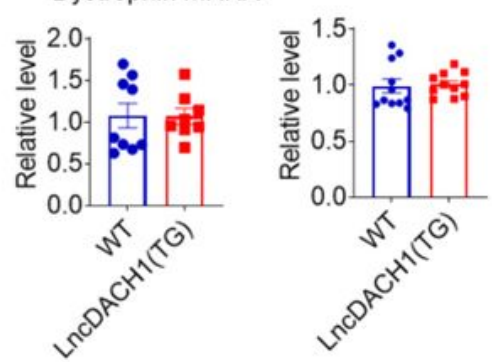

G
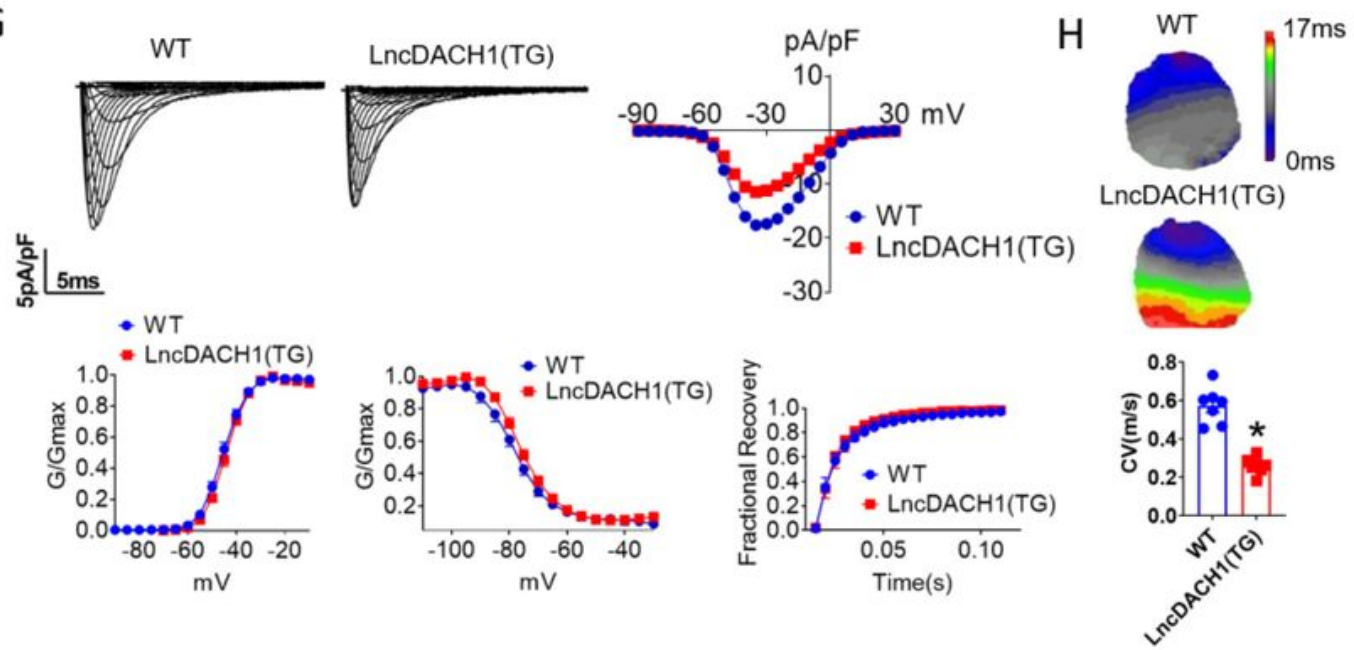

\section{Figure 1}

Binding of IncDACH1 to dystrophin and the effects of cardiomyocyte-specific transgenic overexpression of IncDACH1 (IncDACH1-TG) on the expression and function of sodium channel. (A) Blotting of dystrophin pulled-down by IncDACH1. (B) LncDACH1 precipitated by anti-dystrophin antibody. $\mathrm{N}=4$. * $P<0.05$ vs IgG by one-way ANOVA, followed by Tukey's post-hoc analysis. (C, D) The total, membrane and cytoplasm levels of dystrophin and Nav1.5 by Western blot. N-cadherin is the loading control for 
membrane extracts. $\mathrm{N}=10-11$ for total protein; $\mathrm{N}=$ 7-14 for membrane protein; $\mathrm{N}=8-14$ for cytoplasm protein. ${ }^{*} \mathrm{P}<0.05$ versus WT group. ${ }^{*} \mathrm{P}<0.05$ versus $\mathrm{WT}$ group. $\mathrm{P}$-values were determined by unpaired $\mathrm{t}$ test. (E) Distribution of IncDACH1, dystrophin and Nav1.5 in isolated cardiomyocytes. (F) The mRNA levels of dystrophin and SCN5A. N=8-11. (G) Peak INa currents, I-V curve and kinetics of INa. N=9-15 cells from 3 or more mice. $(\mathrm{H})$ Conduction velocity of perfused hearts by optical mapping recordings. $N=7 .{ }^{*} \mathrm{P}<0.05$ versus WT group. P-values were determined by unpaired $t$ test.

A LnCDACH1 B
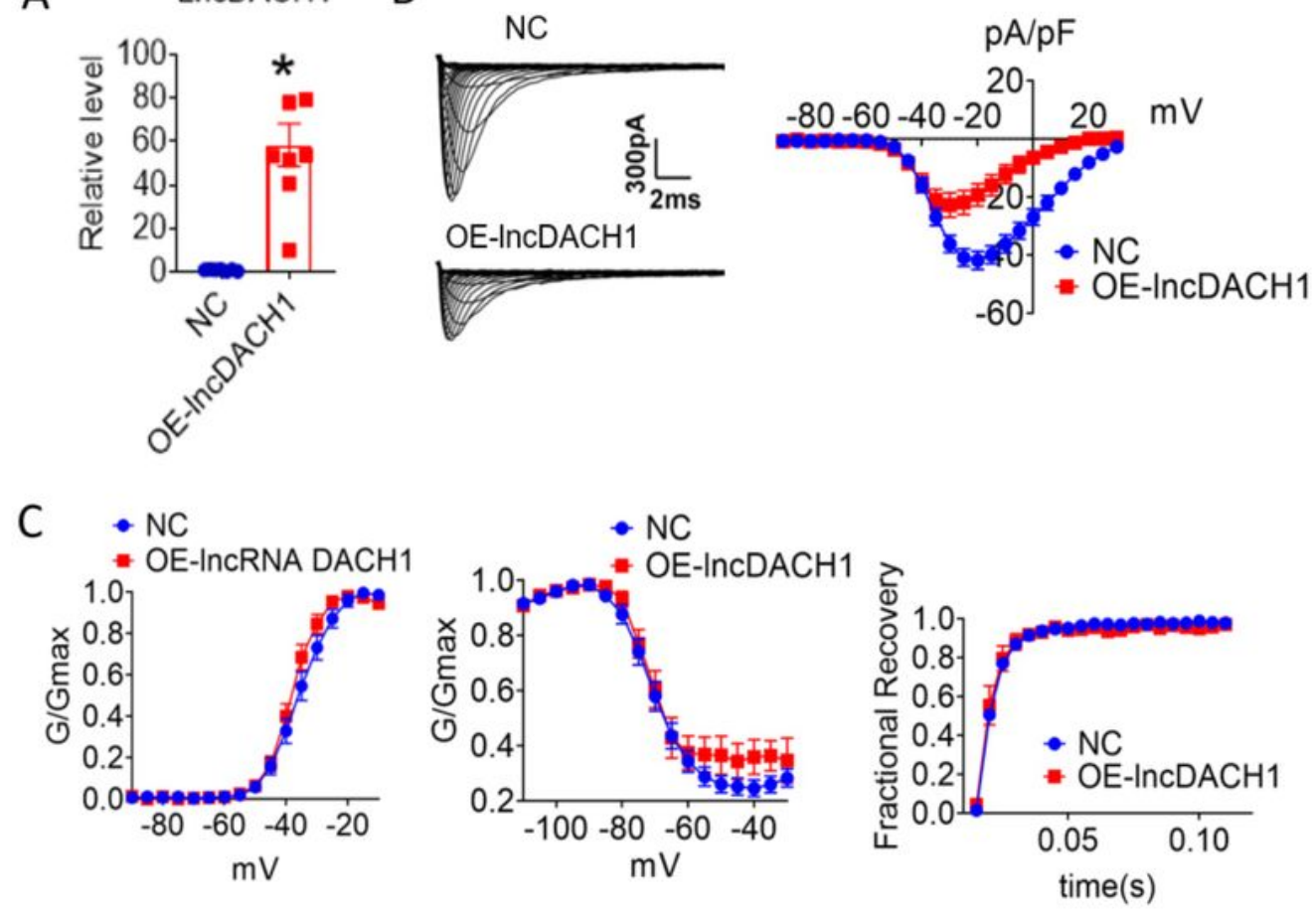

D

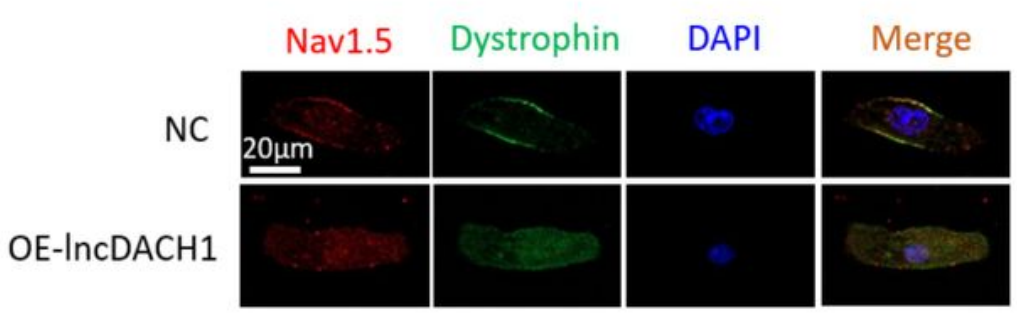

$\mathrm{E}$

SCN5A mRNA Dystrophin mRNA
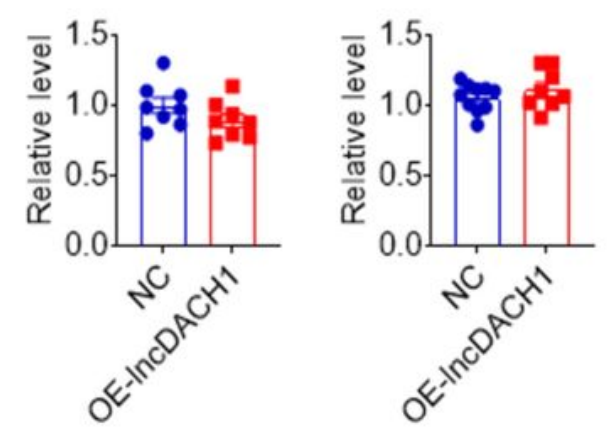

Figure 2 
Effects of IncDACH1 overexpression on sodium channel expression and function in cultured neonatal cardiomyocytes. (A) Verification of the expression of IncDACH1 after transfection of adenovirus carrying IncDACH1. N=7-8 from 3 independent cultures. ${ }^{*} \mathrm{P}<0.05$ versus NC (negative control, empty plasmid). $\mathrm{P}$ values were determined by unpaired t test. $(B, C)$ Peak INa currents, I-V curves and kinetics of INa. $N=12-$ 16 cells from 3 independent cultures. (D) Distribution of Nav1.5 and dystrophin by immunofluorescent staining. (E) The mRNA levels of dystrophin and SCN5A. N=8-10 from 3 independent cultures.

A

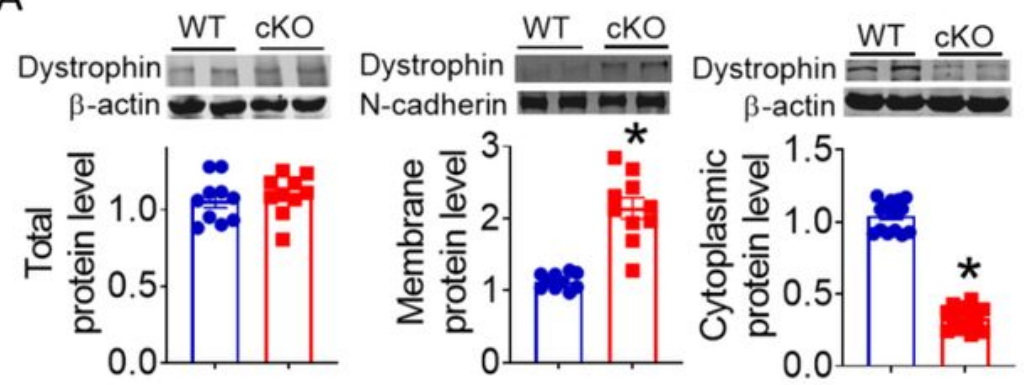

Dystrophin mRNA

B
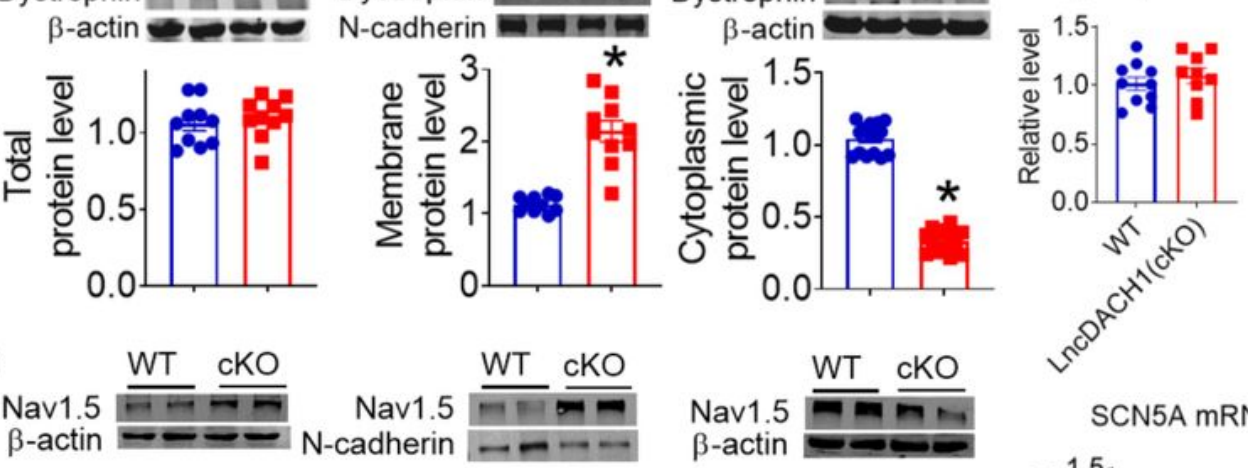

$\beta$-actin $=-\mathrm{N}$-cadherin

SCN5A mRNA
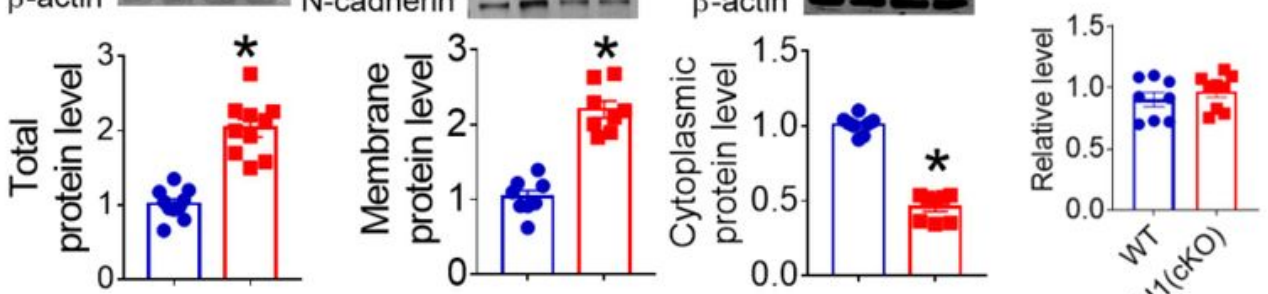

C

Nav1.5 Dystrophin LncDACH1 DAPI Merge

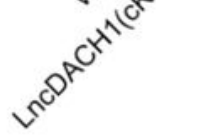

WT
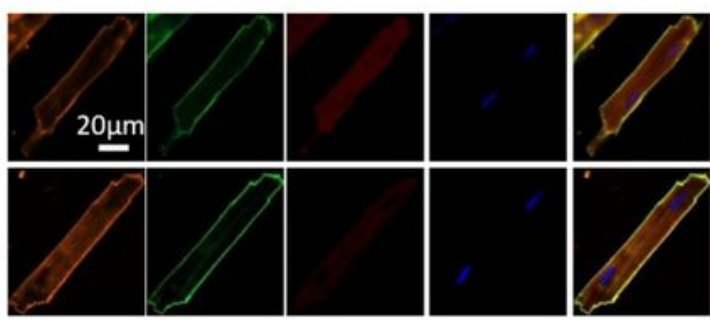

D
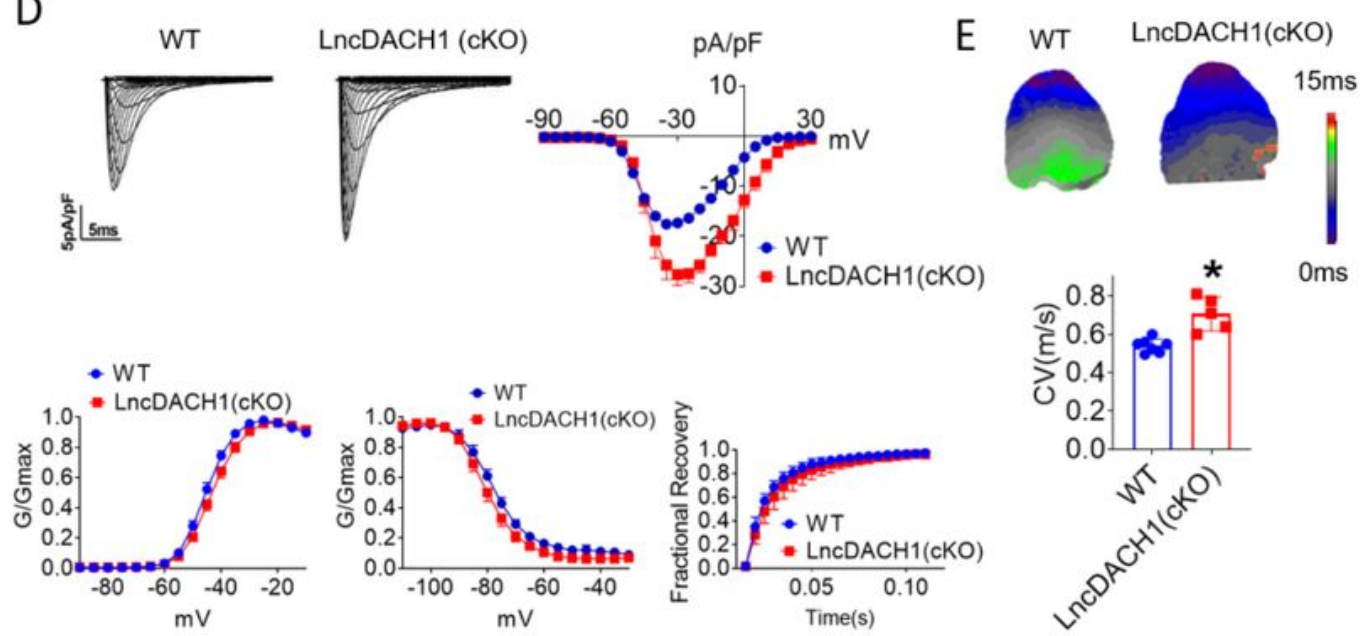

Figure 3 
Conditional knockout of IncDACH1(IncDACH1-cKO) in cardiomyocytes increased peak sodium current, membrane Nav1.5 expression. (A) The total, membrane and cytoplasm levels of dystrophin by Western blot and dystrophin mRNA by qRT-PCR. N-cadherin is the loading control for membrane extracts. $\mathrm{N}=10$ for total protein; $\mathrm{N}=10$ for membrane protein; $\mathrm{N}=15$ for cytoplasm protein. ${ }^{*} \mathrm{P}<0.05$ versus $\mathrm{WT}$ group. $\mathrm{P}$ values were determined by unpaired $t$ test. (B) The total, membrane and cytoplasm levels of Nav1.5 by Western blot and SCN5A mRNA by qRT-PCR. N-cadherin is the loading control for membrane extracts. $\mathrm{N}=10$ for total protein; $\mathrm{N}=8$ for membrane protein; $\mathrm{N}=8$ for cytoplasm protein. ${ }^{*} \mathrm{P}<0.05$ versus WT group. P-values were determined by unpaired t test. (C) Distribution of IncDACH1, dystrophin and Nav1.5 in isolated cardiomyocytes. (D) Peak INa currents, I-V curve and kinetics of INa. $\mathrm{N}=10-15$ cells from 3 or more mice. (E) Conduction velocity of perfused hearts by optical mapping recordings. $N=7$ and 5 . * $P<0.05$ versus WT group. P-values were determined by unpaired $t$ test. 
A LncDACH1 B
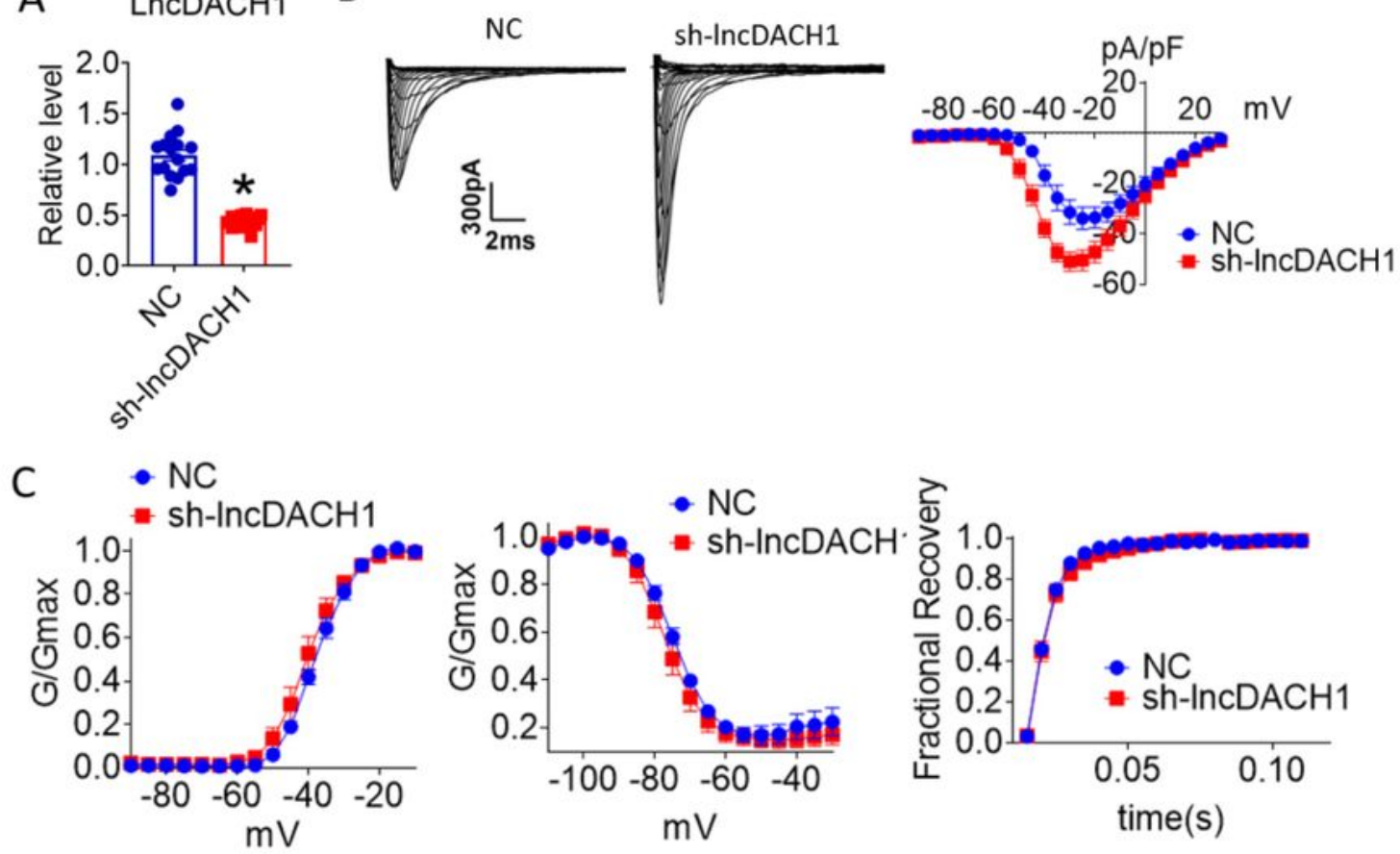

D

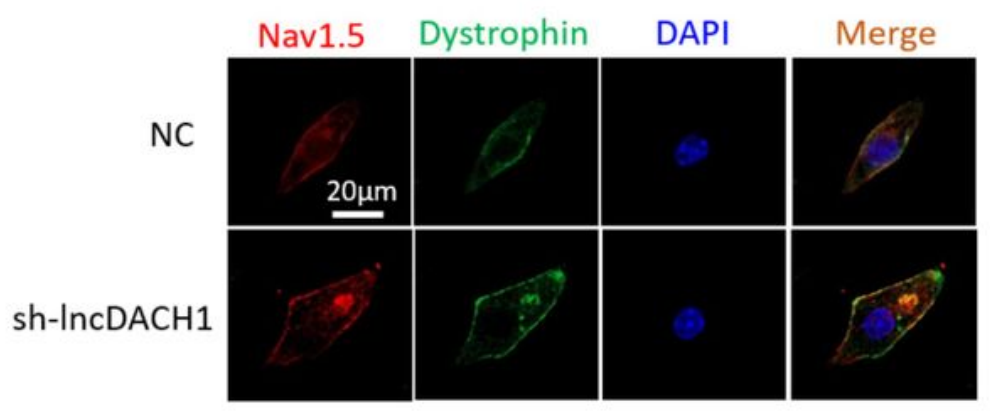

$\mathrm{E}$

Dystrophin mRNA

SCN5A mRNA
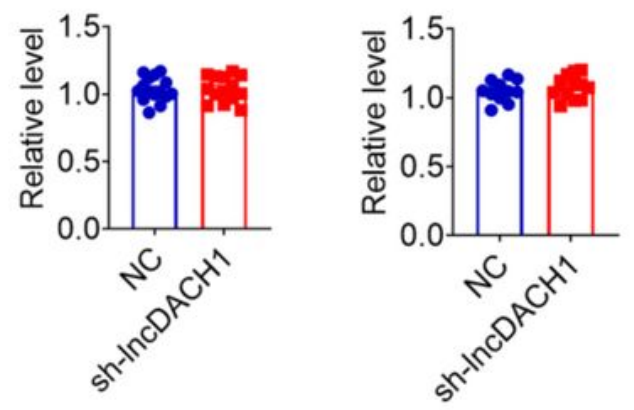

\section{Figure 4}

Effects of IncDACH1 knockdown on sodium channel expression and function in cultured neonatal cardiomyocytes. (A) Verification of the expression of IncDACH1 after infection of adenovirus carrying IncDACH1 siRNAs. $\mathrm{N}=15$ from 3 independent cultures. ${ }^{*} \mathrm{P}<0.05$ versus $\mathrm{NC}$ (negative control, empty plasmids). P-values were determined by unpaired t test. $(B, C)$ Peak INa currents, I-V curve and kinetics of INa. N=9-15 from 3 independent cultures. (D) Distribution of Nav1.5 and dystrophin by 
immunofluorescent staining. (E) The mRNA levels of dystrophin and SCN5A. N=11-15 from 3 independent cultures.

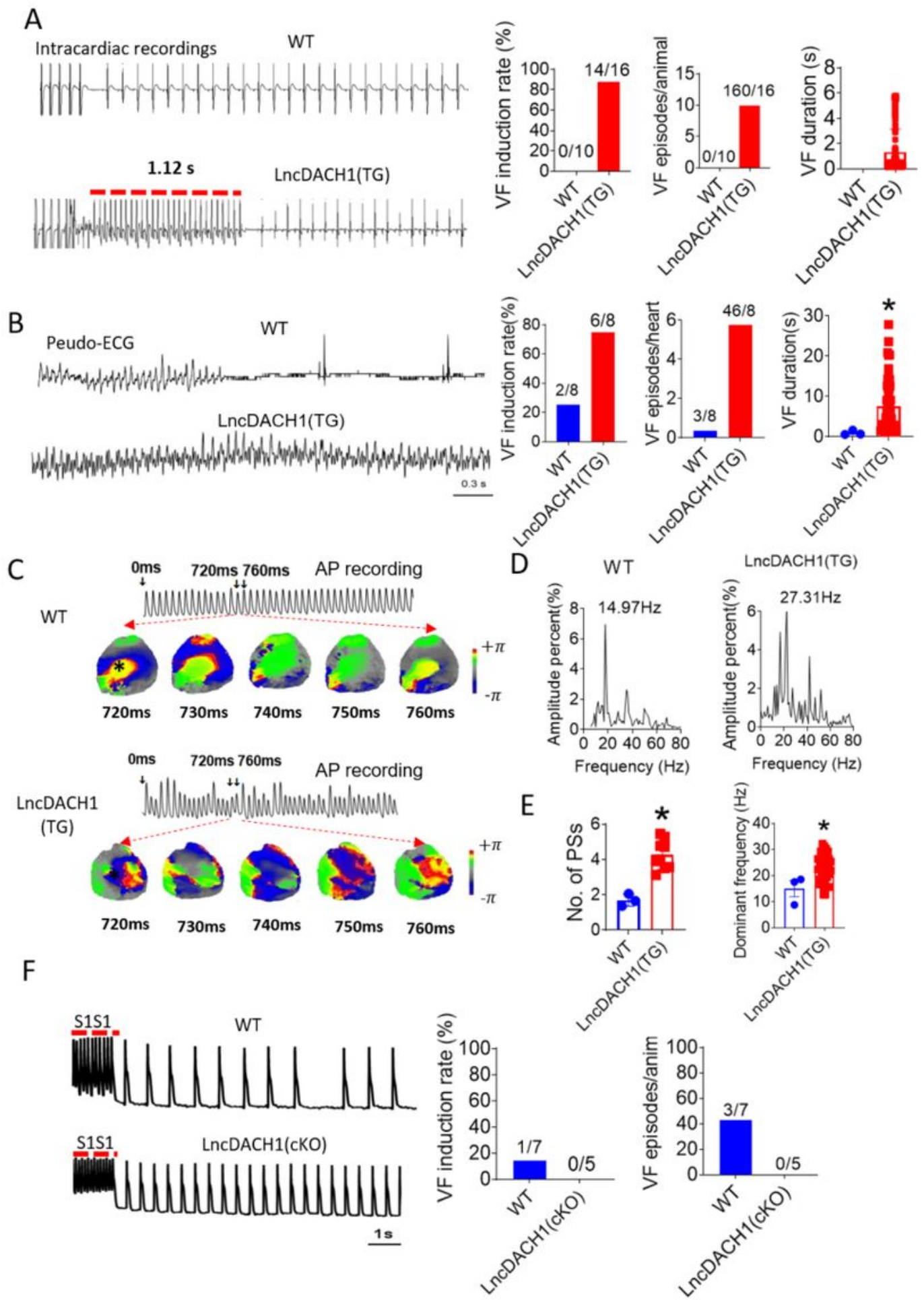

Figure 5

Increased arrhythmia susceptibility in IncDACH1-TG mice. (A) Ventricular fibrillation (VF) induced by S1S2 pacing in intact mice. $\mathrm{N}=10-16$.(B) Ventricular fibrillation (VF) induced by S1S1 pacing of perfused hearts.(C) Break points during VT of WT and IncDACH1-TG mice by optical mapping. Consecutive phase 
maps sampled at 10-ms interval during VF from WT and TG mice. Phase singularities (wavebreaks) are indicated by phase maps. Upper panels showed corresponding optical recording of $\mathrm{VF}$ at asterisk site. $n=8$. (D) Representative of dorminant frequency of VF from WT and TG mice. (E) The number of phase singularities and dorminant frequency of WT and TG mice. (F) Ventricular fibrillation (VF) induced by S1S1 pacing in perfused hearts. $\mathrm{N}=5-7$ mice.

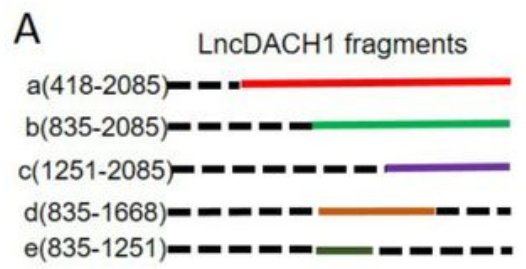

C

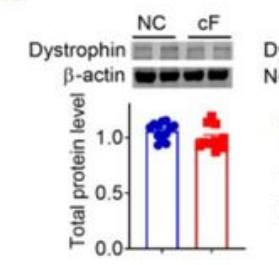

D

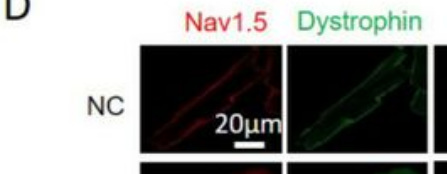

cF.

IncDACH1
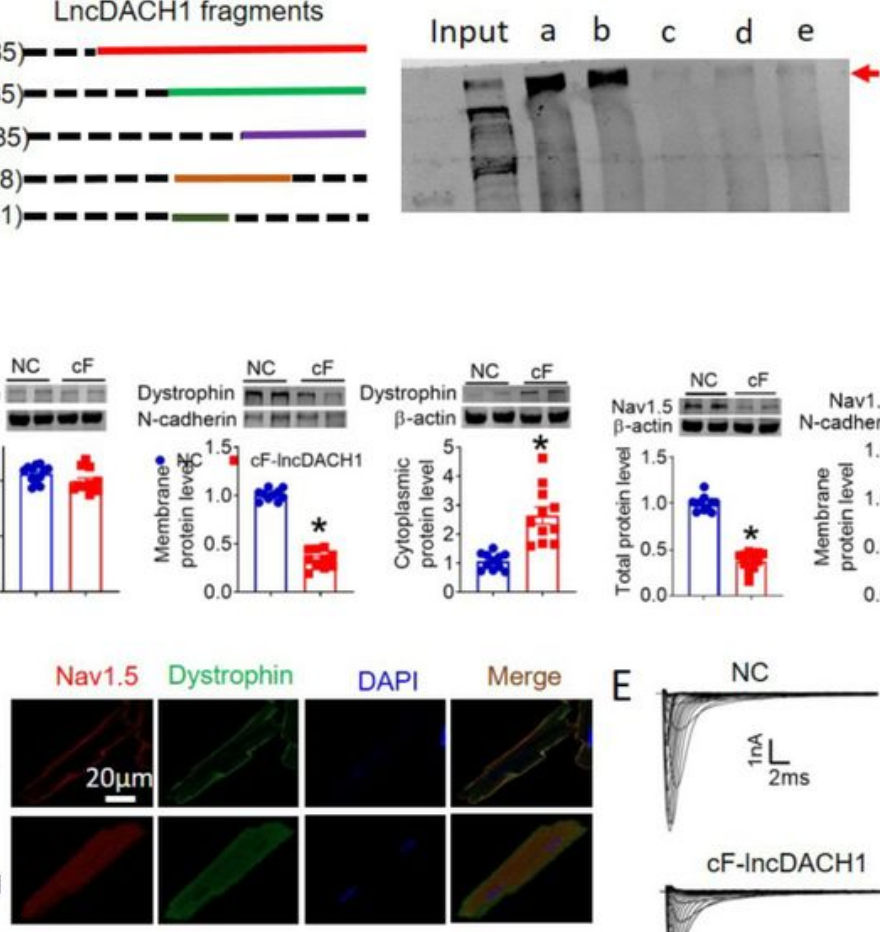

B $\quad \mathrm{CF}-\mathrm{InCDACH} 1$
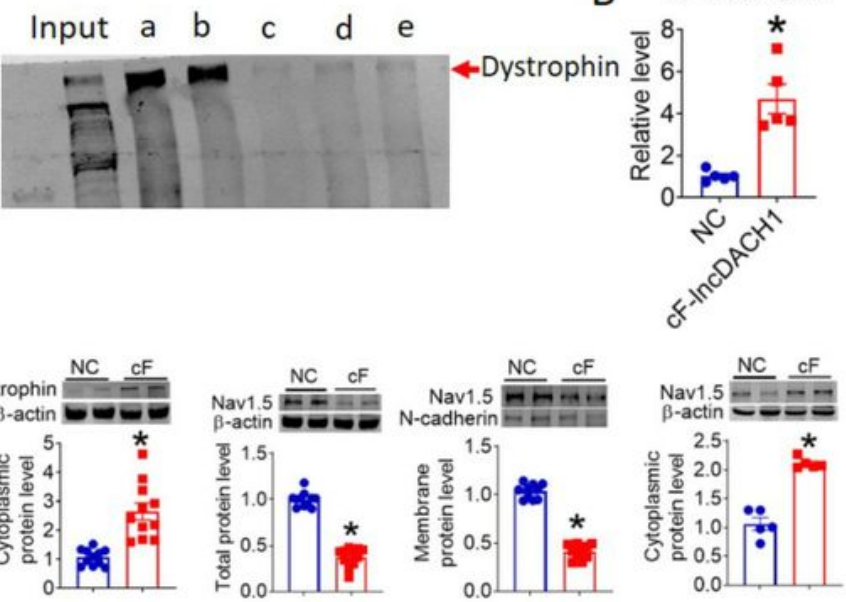

$\mathrm{F}$
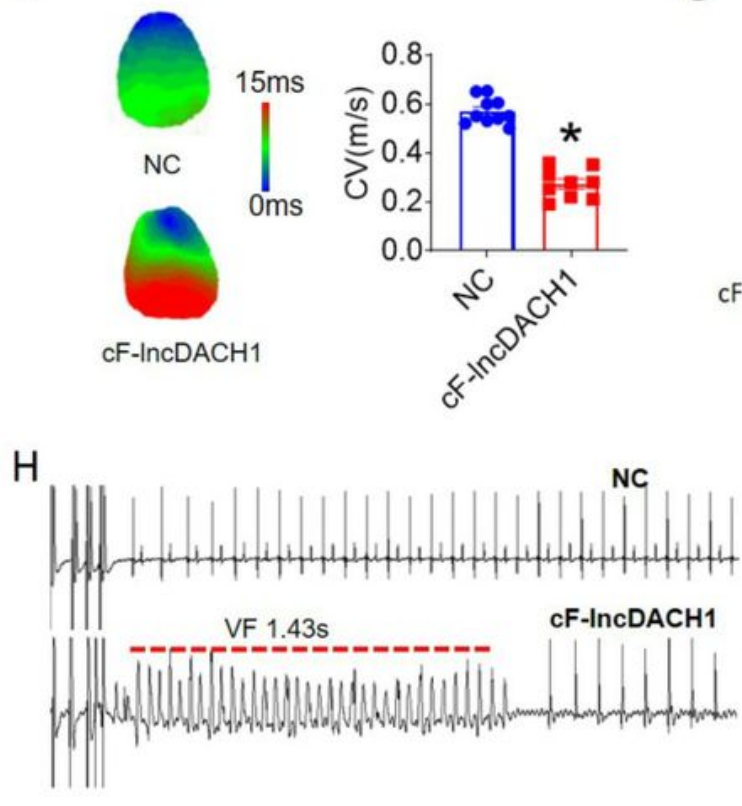

G
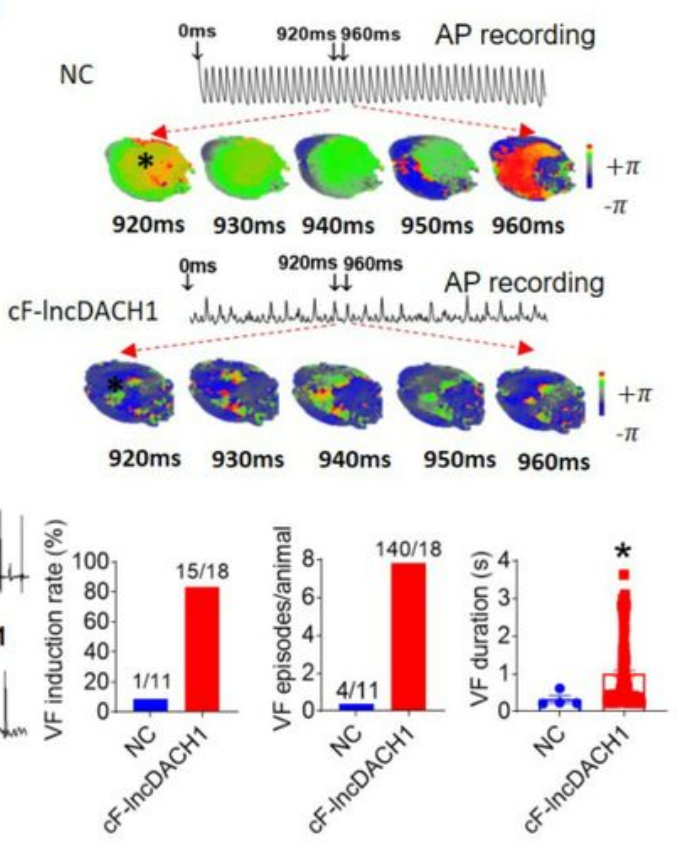

Figure 6 
The conserved fragment of IncDACH1 (cF-IncDACH1) inhibited sodium channel function in mice. (A) Pulldown of dystrophin by fragments of IncDACH1 as indicated. (B) Verification of the expression of cFIncDACH1 after injection of adeno-virus carrying cF-IncDACH1. N=5. ${ }^{*}<0.05$ versus $\mathrm{NC}$ (negative control, Adeno-virus carrying empty plasmid). P-values were determined by unpaired $t$ test. (C) The total, membrane and cytoplasm levels of dystrophin and Nav1.5 by Western blot. $\mathrm{N}$-cadherin is the loading control for membrane extracts. $\mathrm{N}=11-12$ for total protein; $\mathrm{N}=10$ for membrane protein; $\mathrm{N}=5-11$ for cytoplasm protein. ${ }^{*} \mathrm{P}<0.05$ versus $\mathrm{NC}$ group. $\mathrm{P}$-values were determined by unpaired $t$ test. (D) Distribution of dystrophin and Nav1.5 in isolated cardiomyocytes. (E) Representative traces and I-V curve of peak INa currents. $\mathrm{N}=12-15$ from 3 or more mice. ( $F$ ) Conduction velocity of perfused hearts by optical mapping recordings. $\mathrm{N}=9-10$. ${ }^{*} \mathrm{P}<0.05$ versus $\mathrm{NC}$ group. $\mathrm{P}$-values were determined by unpaired $t$ test. $(\mathrm{G})$ Break points during VT by optical mapping. H. Ventricular (VF) induced by S1S2 pacing in intact mice. 
A

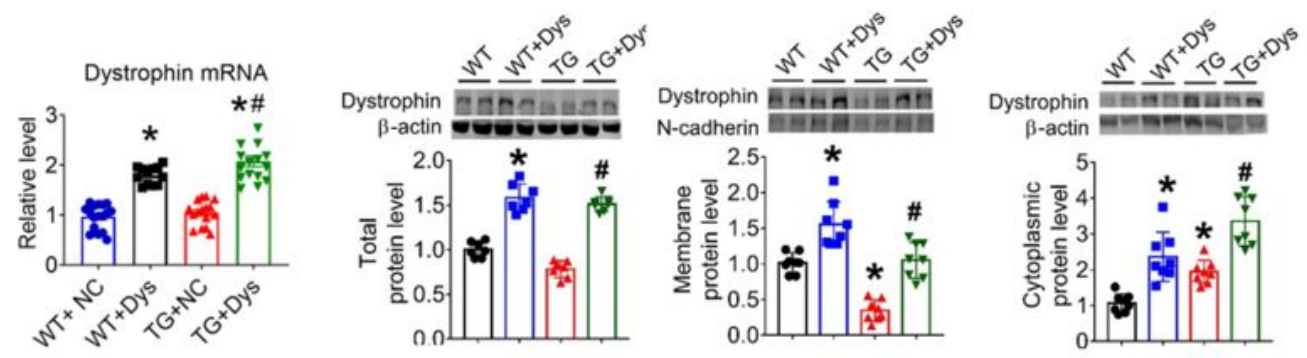

B
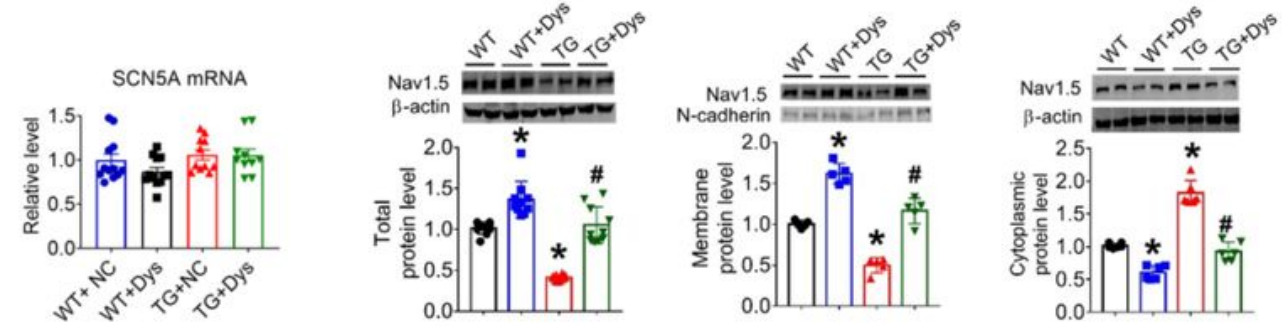

C
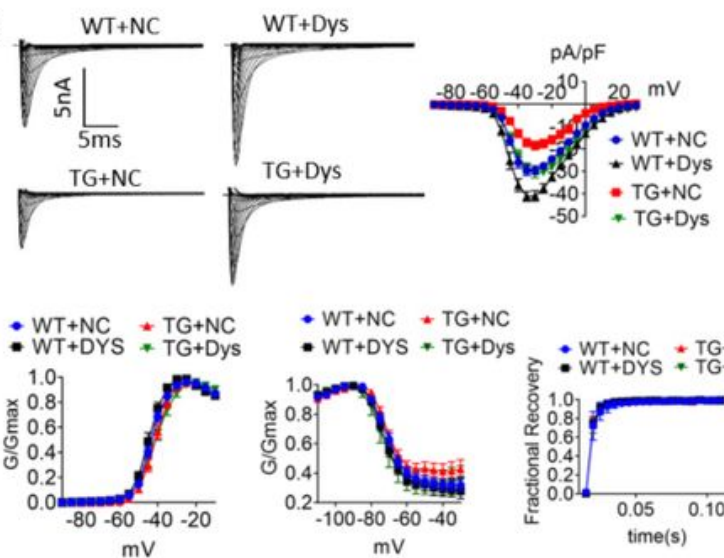

D

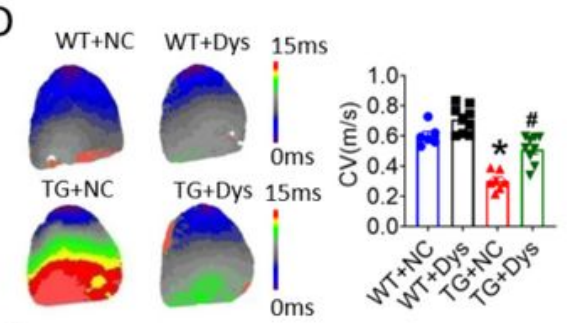

$\mathrm{F}$
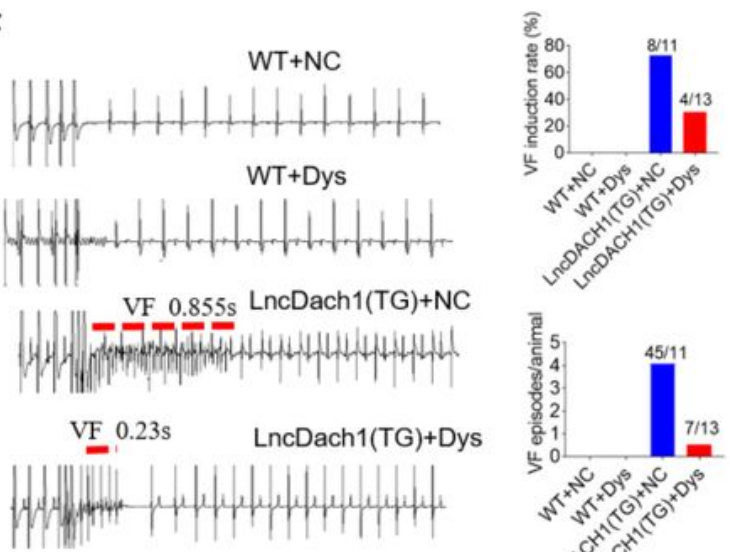

$\mathrm{E}$
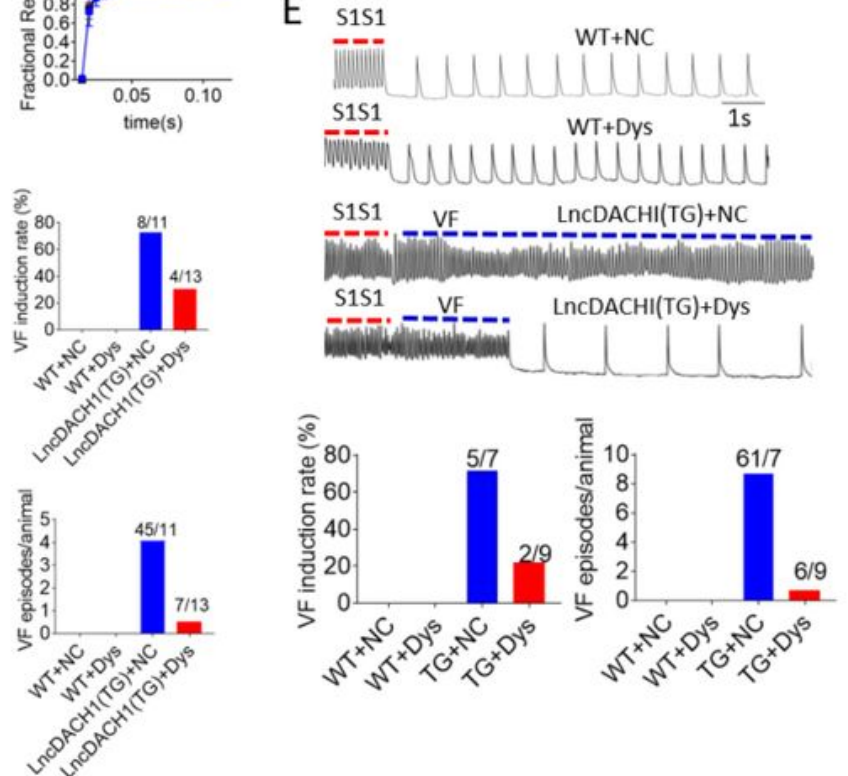

\section{Figure 7}

Activation of dystrophin transcription by AAV9 virus carrying dCas9-SAM system (AAV9-Dys-Act) rescued the remodeling of sodium channel in IncDACH1-TG mice. (A) The mRNA level of dystrophin by real-time PCR ( $\mathrm{N}=12-17)$ and the total, membrane and cytoplasm protein levels of dystrophin by western blot. $\mathrm{N}$ cadherin is the loading control for membrane extracts. $\mathrm{N}=7$ for total protein; $\mathrm{N}=8$ for membrane protein; $\mathrm{N}=8$ for cytoplasm protein. ${ }^{*} \mathrm{P}<0.05$ versus NC group. NC, AAV9 virus carrying dCas9-SAM system with 
control sgRNA; Dys, AAV9 virus carrying dCas9-SAM system with sgRNA targeting dystrophin promoter. (B) The mRNA level of Nav1.5 by real-time PCR $(\mathrm{N}=10-12)$ and the total, membrane and cytoplasm protein levels of Nav1.5 by western blot. $\mathrm{N}$-cadherin is the loading control for membrane extracts. $\mathrm{N}=10$ for total protein; $\mathrm{N}=5$ for membrane protein; $\mathrm{N}=6$ for cytoplasm protein. ${ }^{*} \mathrm{P}<0.05$ versus $\mathrm{NC}$ group. (C) Representative traces, I-V curves and kinetics of peak INa currents. $\mathrm{N}=11-20$ cells from 3 or more mice. (D) Conduction velocity of perfused hearts by optical mapping recordings. $N=6-9$. ${ }^{*} P<0.05$ versus NC group. P-values were determined by unpaired $t$ test. (E) Ventricular fibrillation (VF) induced by S1S1 pacing in perfused hearts. $N=7-10$. (F) Ventricular fibrillation (VF) induced by S1S2 pacing in intact mice. $N=10-13$. The data are analyzed by one-way ANOVA followed by Tukey's post-hoc analysis.

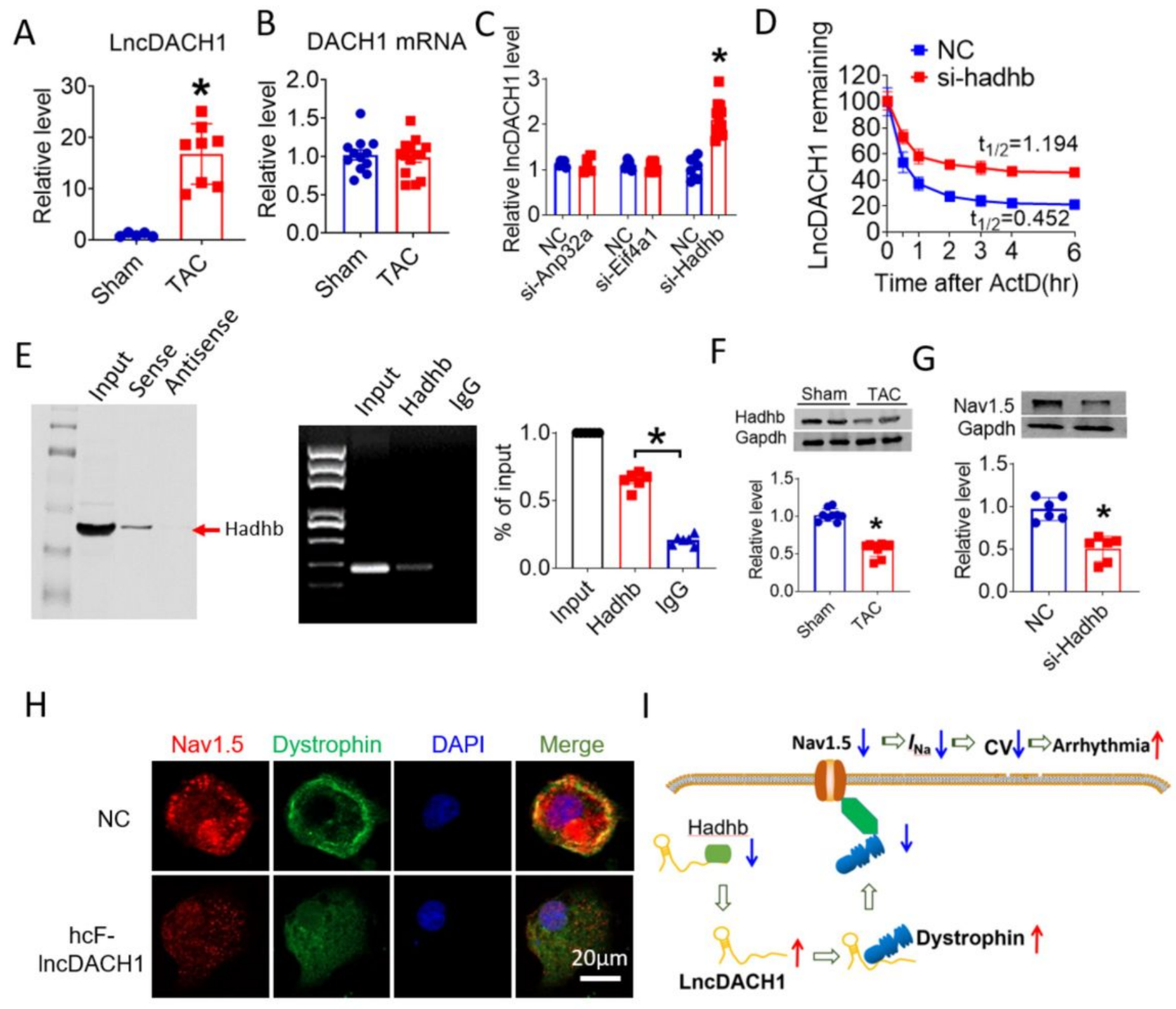




\section{Figure 8}

Hadhb binds to IncDACH1 and promotes its decay. (A) The expression level of IncDACH1 in the hearts of TAC mice. $N=5-8 .{ }^{*} \mathrm{P}<0.05$ by unpaired $t$ test. (B) The mRNA level of $\mathrm{DACH} 1$ in the hearts of TAC mice. $\mathrm{N}=12-14$. (C) Effects of siRNAs for anp32a, eif4a1 and hadhb on the expression of IncDACH1. N=5-12 from 3 independent cultures. ${ }^{*} P<0.05$ by unpaired $t$ test. (D) The effects of hadhb siRNA on the decay of IncDACH1. N=6-15 from 3 independent cultures. (E) The effects of hadhb siRNA on the protein expression of Nav1.5 and dystrophin. (F) Blotting of hadhb pulled-down by IncDACH1, and precipitation of IncDACH1 by anti-hadhb antibody. $N=4$. * $P<0.05$ vs IgG by one-way ANOVA followed by Tukey's post-hoc analysis.

(G) LncDACH1 inhibits Nav1.5 in human iPS differentiated cardiomyocytes. I. Schematic summary of the signaling pathway of hadhb/IncDACH1/dystrophin/Nav1.5.

\section{Supplementary Files}

This is a list of supplementary files associated with this preprint. Click to download.

- supplementalmaterials.docx 